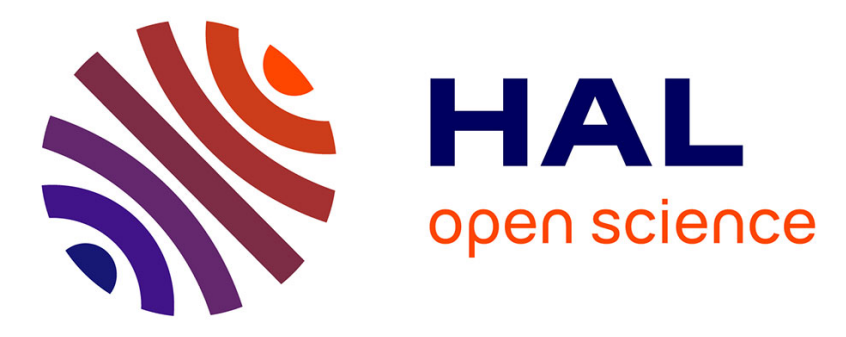

\title{
Modeling the Mullins effect in elastomers swollen by palm biodiesel
}

Andri Andriyana, Mei Sze Loo, Grégory Chagnon, Erwan Verron, Shiau Ying Ch'ng

\section{- To cite this version:}

Andri Andriyana, Mei Sze Loo, Grégory Chagnon, Erwan Verron, Shiau Ying Ch'ng. Modeling the Mullins effect in elastomers swollen by palm biodiesel. International Journal of Engineering Science, 2015, 95, pp.1-22. 10.1016/j.ijengsci.2015.06.005 . hal-01480519

\section{HAL Id: hal-01480519 https://hal.science/hal-01480519}

Submitted on 1 Mar 2017

HAL is a multi-disciplinary open access archive for the deposit and dissemination of scientific research documents, whether they are published or not. The documents may come from teaching and research institutions in France or abroad, or from public or private research centers.
L'archive ouverte pluridisciplinaire HAL, est destinée au dépôt et à la diffusion de documents scientifiques de niveau recherche, publiés ou non, émanant des établissements d'enseignement et de recherche français ou étrangers, des laboratoires publics ou privés.

\section{(c)(1)}

Distributed under a Creative Commons Attribution| 4.0 International License 


\title{
Modeling the Mullins effect in elastomers swollen by palm
}

\section{biodiesel}

\author{
Andri Andriyana ${ }^{a}$, Mei Sze Loo ${ }^{a}$, Grégory Chagnon ${ }^{\mathrm{b}}$, Erwan Verron ${ }^{\mathrm{c}}$, Shiau Ying Ch'ng ${ }^{\mathrm{d}}$
}

\footnotetext{
${ }^{a}$ Mechanics of Soft Materials Laboratory, Department of Mechanical Engineering, Faculty of Engineering, University of Malaya, 50603 Kuala Lumpur, Malaysia ${ }^{\mathrm{b}}$ UJF-Grenoble1/CNRS/TIMC-IMAG UMR5525, Grenoble, France

${ }^{c}$ LUNAM Université, École Centrale de Nantes, GeM, UMR CNRS 6183, BP 92101, 44321 Nantes, France

${ }^{\mathrm{d}}$ Taylor's University, Taylor's Lakeside Campus, Subang Jaya 47500, Selangor Darul Ehsan, Malaysia
}

\begin{abstract}
In the present paper, experimental investigation and continuum mechanical modeling of Mullins effect in swollen elastomers, due to exposure to palm biodiesel, under cyclic loading conditions are addressed. To this end, the nature of Mullins effect in both dry and swollen elastomers is explored and compared. It is found that swelling reduces Mullins effect. Based on experimental observations, in order to account for swelling in the modeling of Mullins effect, two constitutive equations widely used in literature are considered and phenomenologically extended: Continuum Damage Mechanics model and Pseudo-Elastic model. The efficiency of the two extended models are assessed and perspectives for further development are drawn.
\end{abstract}

Keywords:

Constitutive modeling

Elastomer

Mullins effect

Cyclic loading

Swelling

Biodiesel

\section{Introduction}

Elastomers have distinguished themselves among polymeric materials with their own particular characteristics. They have become an almost irreplaceable important part in wide industrial applications in particular automotive components. These applications include vibration isolators, sealing system, flexible piping, gaskets and impact bumpers. During the service, these components are frequently subjected to fluctuating mechanical loading which could lead to fatigue failure (Mars \& Fatemi, 2002; Andriyana, Saintier, \& Verron, 2010b).

Under cyclic loading, rubber materials exhibit strong inelastic responses such as hysteresis, permanent set and stress-softening. The hysteresis may be attributed to viscoleasticity (Bergström \& Boyce, 1998), viscoplasticity (Lion, 1997) or to strain-induced crystallization (Trabelsi, Albouy, \& Rault, 2003). The stress-softening corresponds to a change of the mechanical properties after the material has been subjected to a deformation. More precisely, it manifests as a non-neglectable loss of stiffness during the transition from the first cycle to the second one. This softening greatly attracts the attention of many researchers after it was reported for the very first time in rubber vulcanizates by Bouasse and Carriére (1903). However, the corresponding stress-softening is often referred to as the Mullins effect after Mullins (1948). The most evident sign that stress-softening has taken place can be concluded from the stress-strain response where one can observe that a lower stress is needed to bear the same strain generated from the first loading phase. While stress-softening is 
commonly observed in filled rubbers and unfilled crystallized rubbers, no study has reported its occurrence in an unfilled uncrystallized rubber (Diani, Fayolle, \& Gilormini, 2009).

As reviewed by Diani et al. (2009), there are many efforts in proposing different theories to explain the Mullins effect in dry rubber. Nevertheless, no unanimous microscopic explanation for this softening is available up to this date (Marckmann et al., 2002; Diani et al., 2009). The first attempt to describe the Mullins effect is through a phenomenological approach. Mullins and Tobin (1957) proposed that rubber initially contains both hard and soft phases. During deformation process, the hard phase transforms into the soft one. Their theory was successfully adopted in a number of works (Wineman \& Huntley, 1994; Huntley, Wineman, \& Rajagopal, 1996; Huntley, Wineman, \& Rajagopal, 1997; Beatty \& Krishnaswamy, 2000; Qi \& Boyce, 2004). Simo (1987) adopted the concept of Continuum Damage Mechanics (CDM) where Mullins effect was considered as a damage phenomenon and was described by a scalar damage parameter. Thus, the material response is characterized by multiplying the classical hyperelastic strain energy with a reducing parameter representing damage level. Different forms of damage parameter were proposed in the literature (Miehe, 1995; Ogden \& Roxburgh, 1999; Miehe \& Keck, 2000; Chagnon, Verron, Gornet, Marckmann, \& Charrier, 2004). In contrast to Miehe (1995) and Chagnon et al. (2004) who assumed that damage evolves when the applied level of deformation is undergone by the material for the first time, Ogden and Roxburgh (1999) proposed that damage stays zero when the material is subjected to a level of deformation never applied, and evolves in the range of submaximal deformation. The latter is known as the Pseudo-Elastic (PE) model.

The second approach is through physical interpretation (Govindjee \& Simo, 1991; Killian, Strauss, \& Hamm, 1994; Klüppel \& Schramm, 9:742-754; Marckmann et al., 2002; Freund, Lorenz, Juhre, Ihlemann, \& Klüppel, 2011). Marckmann et al. (2002) reported the development of a new network alteration theory to describe the Mullins effect where they considered the Mullins effect as consequence of breakage of links inside the material, involving both filler-matrix and chain interaction links. This new alteration theory was implemented by modifying the eight-chains constitutive equation of Arruda and Boyce (1993). The accuracy of the resulting constitutive equation was demonstrated on cyclic uniaxial experiments for both natural rubbers and synthetic elastomers. Chagnon, Verron, Marckmann, and Gornet (2006) later modified this network alteration theory to include the dangling chains effect in the network and proposed that the number of monomers involved in the elastic response of the material is a decreasing function of the maximum deformation. Further refinements to account for deformation-induced anisotropy in Mullins effect were proposed in the literature. Indeed, as pointed out early by Mullins (1948) and more recently by Laraba-Abbes, Ienny, and Piques (2003), Diani, Brieu, and Gilormini (2006), Itskov, Haberstroh, Ehret, and Vohringer (2006), Itskov, Ehret, Kazakevičiūtè-Makovska, and Weinhold (2010) and Machado, Chagnon, and Favier (2012), the material undergoes significant anisotropic softening due to the application of mechanical loading.

In addition to fluctuating mechanical loading, many industrial rubber components are exposed to aggressive solvents, e.g. in o-rings, hoses and sealing applications (Selvadurai \& Yu, 2006). The exposure to such hostile environment is crucial since it speeds up material degradation in the form of swelling (Flory, 1953; Treloar, 1975). The corresponding degradation in rubber components is a major concern in Malaysia and Indonesia who actively develop palm biodiesel as partial substitution to conventional petroleum fuels (Jayed et al., 2011). Indeed, while the use of palm biodiesel offers an attractive alternative for diesel engines since their use does not require extensive engine modification and is environmentally friendly, it is established that utilizing palm biodiesel in the existing engine system creates several compatibility issues on the automotive rubber components (Haseeb, Jun, Fazal, \& Masjuki, 2011). Thus, the need to characterize and to model the mechanical response of rubber materials under fluctuating mechanical loading taking into consideration swelling due to material exposure to aggressive solvents such as palm biodiesel becomes a critical issue for durability analysis of such engineering components.

In contrast to dry rubbers, only few studies on the observation and modeling of Mullins effect in swollen rubbers or gels are available in the literature (Webber, Creton, Brown, \& Gong, 2007; Lin, Fan, Marcellan, Hourdet, \& Creton, 2010; Andriyana, Chai, Verron, \& Johan, 2012; Chai, Andriyana, Verron, \& Johan, 2013a; Chai, Andriyana, Verron, \& Johan, 2013b). Hence, the objective of the present work is to characterize and to model the Mullins effect in rubber under cyclic loading taking into consideration the swelling. For this purpose, mechanical tests are conducted in order to compare the nature of Mullins effect in dry and swollen rubbers. Moreover, two classical models for Mullins effect in dry rubber widely used in the literature are considered and extended to account for swelling: CDM and PE models. It is to note that the approach used for the development of the model in the present work is different with the one proposed by Chai et al. (2013b). Indeed, our approach is based on the split of the deformation gradient tensor into mechanical and swelling parts. The performance of the two extended CDM and PE models are discussed by comparing their ability to capture Mullins effect in swollen rubbers under cyclic loading.

\section{Experimental program}

\subsection{Materials}

The material investigated is a commercial grade of carbon black-filled nitrile rubber (NBR) with 60 shore hardness and 25 wt\% of carbon-black. Due to confidentiality constraint, the detailed compound ingredients are not provided here. The specimens used in this study are in the form of hollow diabolo having a height, outer diameter, and wall thickness of $55 \mathrm{~mm}, 25 \mathrm{~mm}$, and $6 \mathrm{~mm}$ respectively. The swollen rubber specimens are obtained by immersing dry specimens in palm biodiesel until desired swelling levels are achieved. Three swelling levels are considered: $0 \%, 5 \%$ and $7 \%$ in the basis of volume change, which correspond respectively to no immersion (dry), 1 week and 2 weeks of immersion duration. No standard is followed regarding the choice of the specimens and immersion durations. The detailed features of the specimen and 
chemical compositions of the palm biodiesel used in the present study are provided in our previous works (Chai, Andriyana, Verron, Johan, \& Haseeb, 2011; Ch’ng, Andriyana, Verron, Kahbasi, \& Ahmad, 2013).

\subsection{Mechanical tests}

To investigate the effect of swelling on the mechanical response of rubber in particular the Mullins effect, all mechanical tests were conducted using an Instron tensile testing machine. Three types of specimens were considered: dry (10 specimens), 5\% swollen ( 1 week immersion, 10 specimens) and 7\% swollen ( 2 weeks immersion, 10 specimens). Moreover, four types of mechanical testing were conducted. A constant strain rate of $0.02 \mathrm{~s}^{-1}$ was imposed throughout the testing to avoid any excessive increase of temperature in the specimen, i.e. thermal effects are not considered. The four types of mechanical tests are elaborated as below:

$\triangleright$ Test 1

Monotonic uniaxial tensile tests. The specimens are subjected to an increasing monotonic tension load until they break.

$\triangleright$ Test 2

Cyclic uniaxial tension tests:

(a) The specimens are subjected to cyclic loading up to stretch of 2 .

(b) The specimens are subjected to cyclic loading up to stretch of 4.

Note that the stretch is defined by the ratio between the current length to the initial length of the rubber specimen. In the case of swollen rubbers, the specimen initial length corresponds to the swollen-unstressed length. Both specimens from (a) and (b) are then kept for a month after undergoing the corresponding test. After a month, similar tests are re-conducted on them to investigate the recovery of Mullins effect.

$\triangleright$ Test 3

Cyclic tensile tests with increasing maximum stretch. The stretch increment is 0.5 .

$\triangleright$ Test 4

Aging tests. The dry and swollen specimens are kept for a month in room temperature without undergoing any initial mechanical tests. After a month, the specimens are subjected to monotonic uniaxial tensile test, similarly to Test 1 , thereby allowing the observation of aging effect on the mechanical response.

Remark 1. In the following sections, the swelling in rubber specimens is described in terms of the degree of swelling, denoted $J_{s}$, defined by the ratio between the volume of swollen specimen and the one of the dry specimen. Using this definition, three degrees of swelling are considered in the present study: $J_{s}=1$ (dry), $J_{s}=1.05$ ( $5 \%$ volume change due to 1 week immersion) and $J_{s}=1.07$ ( $7 \%$ volume change due to 2 weeks immersion).

\subsection{Results}

\subsubsection{Stress-stretch response}

The stress-stretch responses of dry and swollen rubbers subjected to simple monotonic tension loading up to fracture (Test 1) are shown in Fig. 1. In the case of swollen rubbers, the engineering stress is defined with respect to

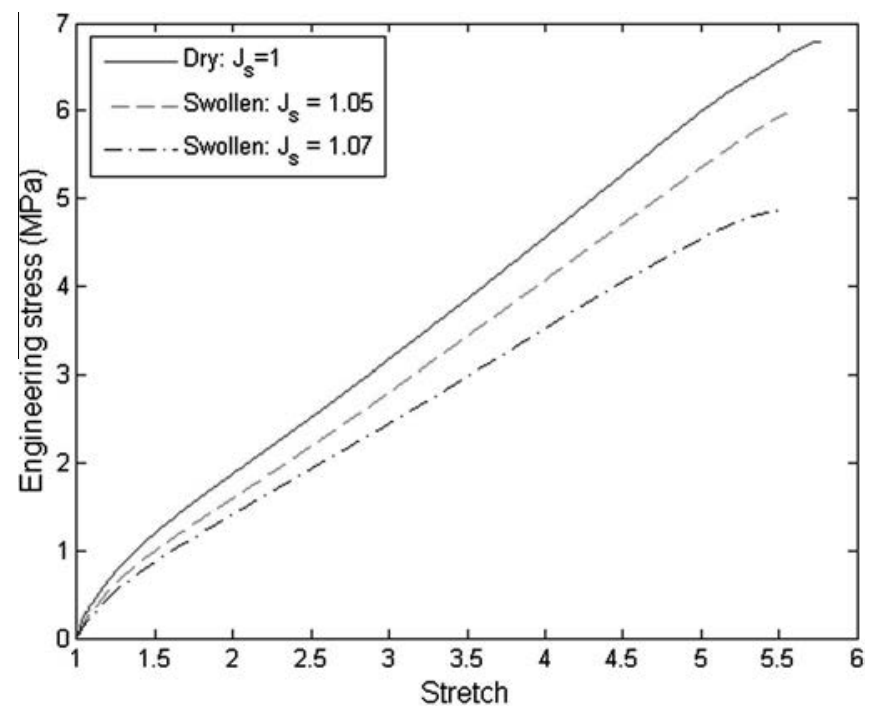

Fig. 1. Stress-stretch curve of materials under monotonic tensile loading (Test 1). 
swollen-unstressed area of specimens. In this figure, no apparent difference is observed in the nature of stress-stretch behaviors between the dry and swollen rubbers. Nevertheless, the stress-stretch responses of swollen rubbers are lower than the dry one since the presence of solvent in rubbers degrades their properties (Flory, 1953; Treloar, 1975; Chai et al., 2011). Moreover, it is observed that the stretch at fracture of swollen rubbers is lower than the one of dry rubber.

The stress-stretch responses of dry and swollen rubbers subjected to five cycles of uniaxial tension up to stretch of 2 and stretch of 4 (Test 2) are presented in Fig. 2. It is clearly shown that both dry and swollen rubbers exhibit inelastic behaviors, i.e. stress-softening due to Mullins effect, hysteresis and permanent set. The Mullins effect is mainly observed between the first and second cycles. Indeed, it is observed that softening which takes place after the second cycle is not significant. Nevertheless, significant hysteresis is still observed even when the material has been softened. Relatively smaller stress-softening and hysteresis are observed in swollen rubbers. The recovery of Mullins effect is discussed in detail in Section 2.3.2.

In order to probe more closely on the effect of swelling on permanent set, the amount of permanent set (from Fig. 2) as a function of number of cycles are plotted in Fig. 3 for the dry and swollen rubbers. In this figure, the permanent set corresponds to the residual stretch, i.e. the value of stretch at zero stress during unloading. It appears that swelling reduces permanent set at all number of cycles for both maximum amounts of stretch.

The stress-stretch responses of dry and swollen rubbers subjected to cyclic uniaxial tension loading with increasing maximum stretch (Test 3) are shown in Fig. 4. The corresponding responses under monotonic tensile test (Test 1) are also included as comparison. Generally, stress-softening features are preserved for all three conditions at different degrees of swelling. Moreover, it is worth to note that the return of the reloading curve approaches nearly to the monotonous curve after being stretched beyond the maximum stretch previously applied. For both dry and swollen rubber, the dependence
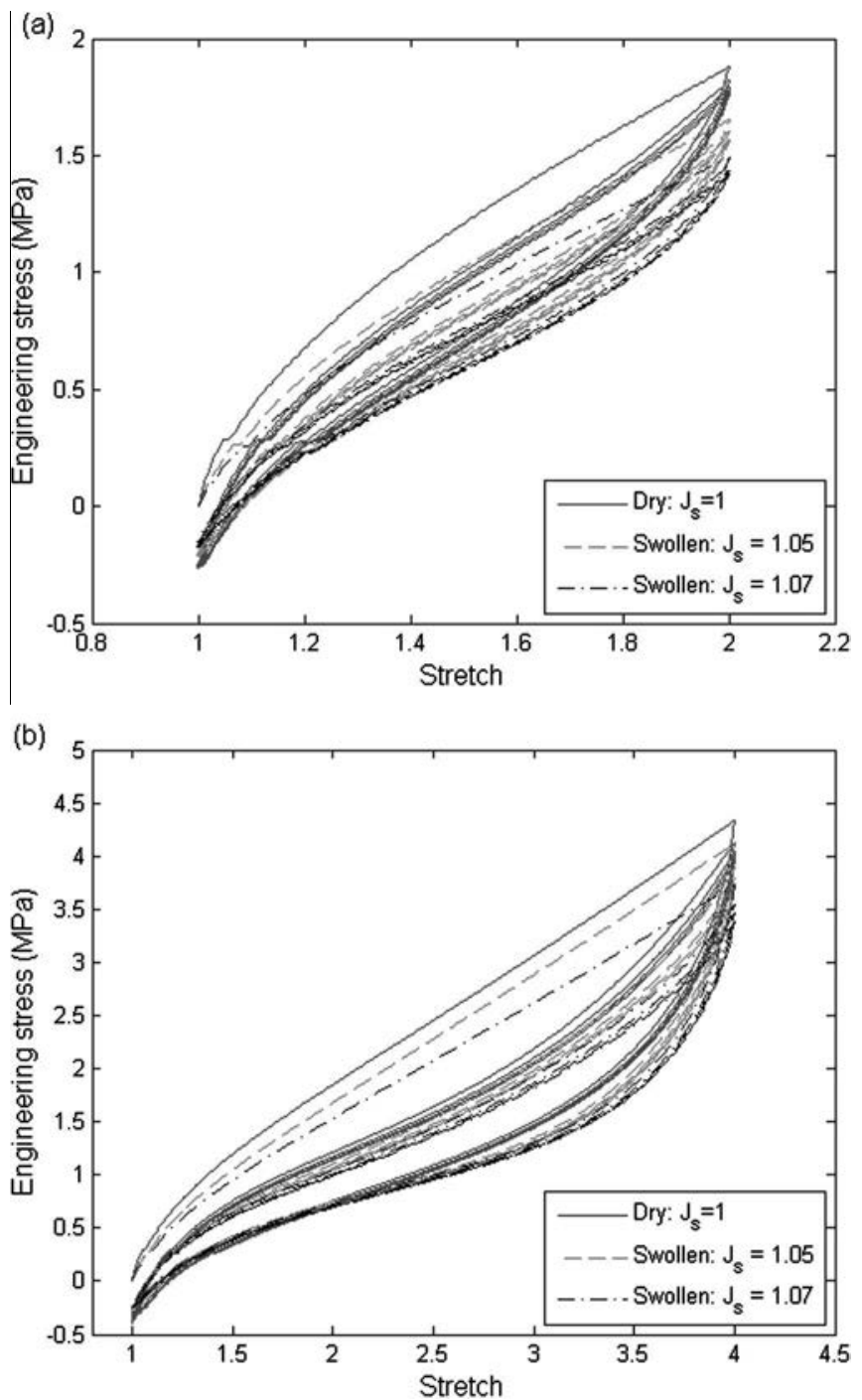

Fig. 2. Stress-stretch curve of materials under 5 cycles of loading (Test 2) at (a) maximum stretch of 2 and (b) maximum stretch of 4. 


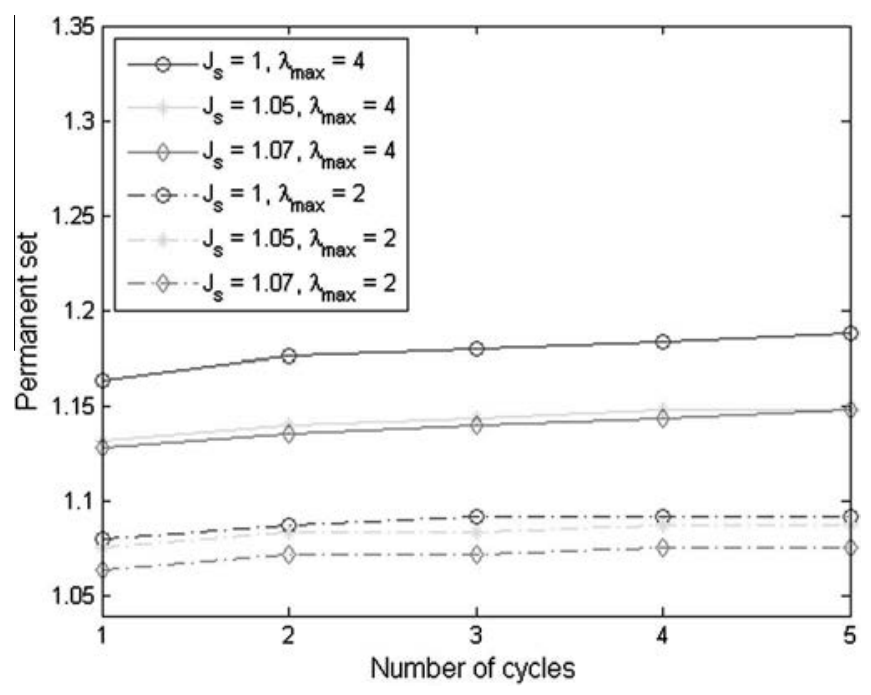

Fig. 3. Evolution of permanent set in dry and swollen rubbers as a function of number of cycles for two values of maximum stretch: 2 and 4 .

of Mullins effect on the maximum deformation previously endured by the materials is clearly shown in this figure. Moreover, they exhibit permanent set which increases as the materials are stretched further. Indeed, independently of the degree of swelling, the value of stretch at zero stress during unloading appears to increase as the maximum stretch in a cycle increases.

The effects of aging on the mechanical responses of dry and swollen elastomeric specimens (Test 4) are depicted in Fig. 5. Here the virgin specimens are subjected to monotonic tensile loading after being kept for one month. Note that for swollen specimens, they are kept after being immersed in the solvent until the desired degree of swelling is achieved. As shown in Fig. 5, it appears that no significant aging effect has taken place in both dry and swollen rubbers. Indeed, after being kept for one month, their mechanical responses are similar to the ones obtained in Test 1 . Thus, the effect of aging on general mechanical responses of materials is not significant. Furthermore, in the recovery of Mullins effect detailed in the next section, aging contribution to the recovery process can be neglected.

\subsubsection{Recovery of Mullins effect}

As shown in Fig. 5, the aging effect can be neglected in the pursuit of further observation in the recovery of Mullins effect. The corresponding recovery in dry and swollen rubber specimens is presented in Fig. 6. In each graph, two curves are plotted. The blue solid line corresponds to the response of rubber specimen during five cycles of loading. The specimen is subsequently kept for one month before undergoing similar test where the result is presented in red dashed line. As indicated in the three graphs of Fig. 6, it appears that the Mullins effect in both dry and swollen rubbers is partially recovered.

To closely observe the effect of swelling on the recovery of Mullins effect, by referring to Fig. 7(a), the following definition is used in the present paper:

$$
\% \text { of recovery }=\frac{\text { Area } \mathrm{OBC}}{\text { Area } \mathrm{OAC}}
$$

where the area of $\mathrm{OBC}$ is the area delimited by the fifth uploading curve of the material before recovery test and first uploading curve of the same material after recovery test. Area OAC is the one delimited by the first and fifth uploading paths of the material before recovery test. It is to recall that the fifth uploading path is assumed to be similar to the second path since it is observed that apart from the first two cycles, the second and the following cycles coincides (see Fig. 6). Moreover, Mullins effect is usually considered between the first and second uploading paths (Diani et al., 2009). The effect of swelling on the recovery of Mullins effect is illustrated in Fig. 7(b) for two values of maximum stretch: 2 and 4. It is observed that swollen rubber specimens experience a greater recovery than the dry one. This occurrence is similar to Harwood and Payne's finding in the recovery of Mullins effect of rubber through swelling (Harwood \& Payne, 1967).

\subsection{Summary}

The results presented in the present section clearly indicated the complexity of the material responses, i.e. Mullins effect, hysteresis and permanent set. Since the focus of the present study is on the Mullins effect, the experimental results must be treated in order to highlight the characteristics of Mullins effect in swollen rubber. Other inelastic responses, i.e. hysteresis and permanent set, are not considered. Moreover, for the sake of simplicity, the recovery of Mullins effect is neglected in the present work. To this end, the following data treatment similar to the one proposed by Chagnon et al. (2004) is adopted which can be summarized as: 

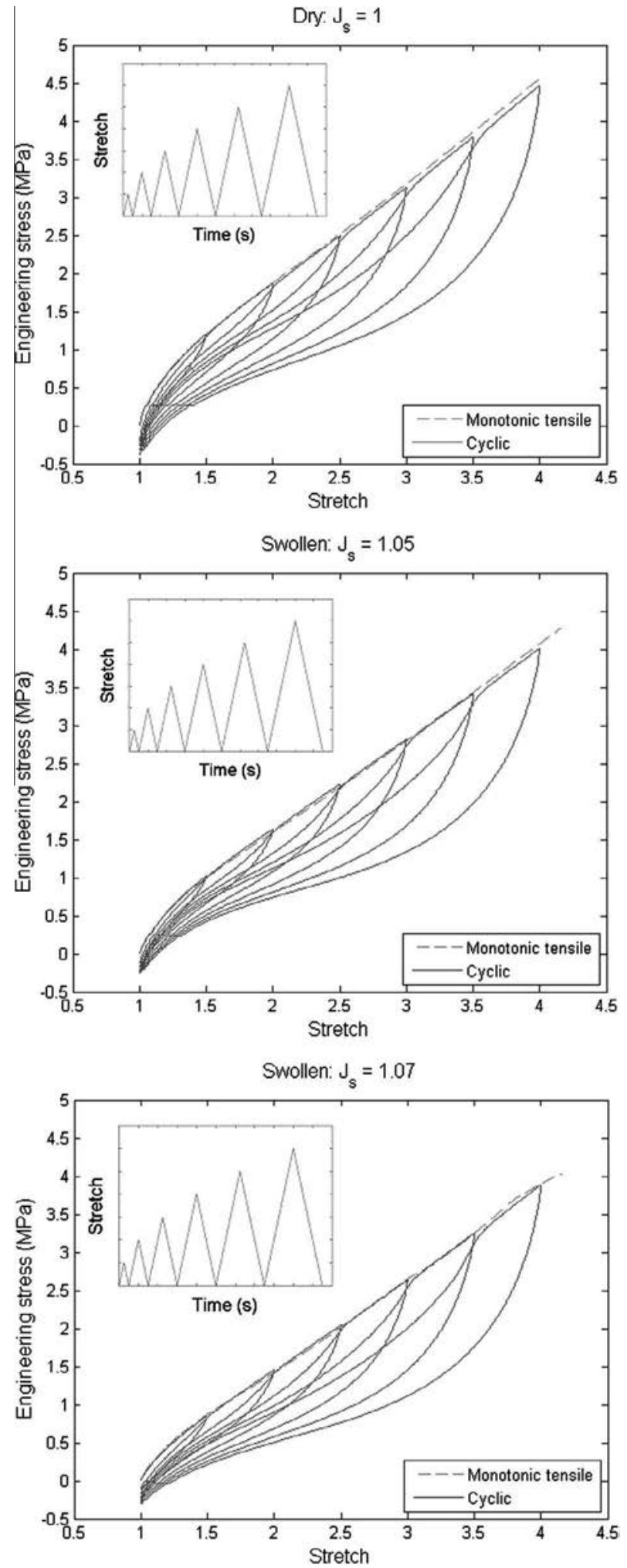

Fig. 4. Material responses under cyclic loading with increasing maximum stretch (Test 3). 


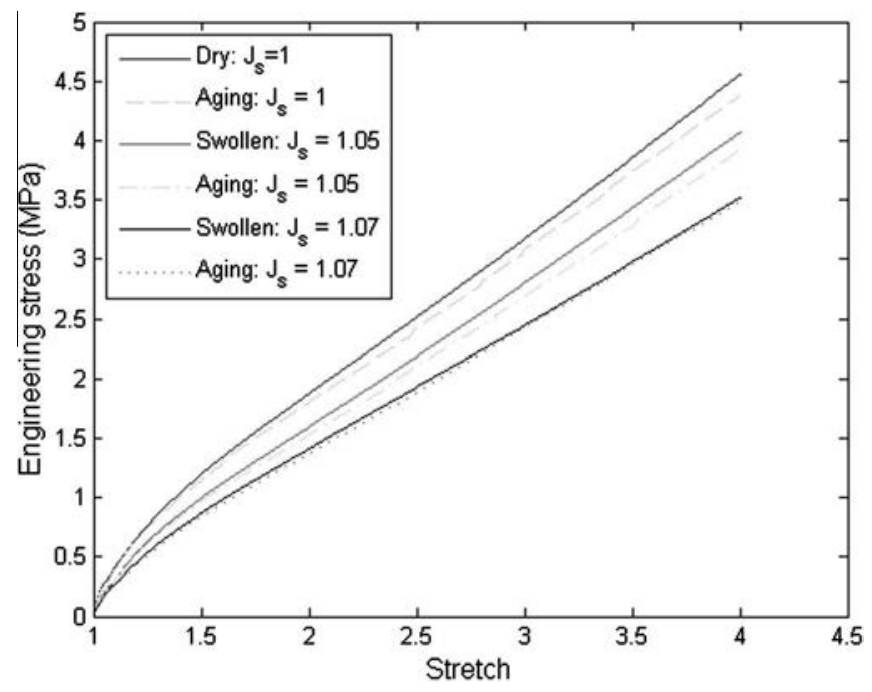

Fig. 5. Comparison of stress-stretch curves of materials before and after aging test (Test 4).

1. The reloading path of the second cycle is assumed to coincide with the downloading path of the first cycle.

2. The unloading data are horizontally shifted such that they start from zero strain (stretch of 1 ), i.e. permanent set is not considered.

3. The unloading data are extended to re-join the primary loading path (obtained from the monotonic tensile test).

The treated experimental results for dry and swollen rubbers are presented in Fig. 8. In this figure, the red dashed line corresponds to the result of monotonic tensile and is referred to as the primary curve. The blue solid lines are referred to as the secondary curves which correspond to the shifted unloading curves so that they start from stretch of one. The data in Fig. 8 will be used as the basis for the discussion on the modelling of Mullins effect in swollen rubber.

Remark 2. According to Chai et al. (2013a), the NBR swollen up to $5 \%$ and $7 \%$ are far from equilibrium swelling. Thus, solvent distribution across cross-sections of the specimen is not homogeneous. As a first approximation, in the following model development, the material is considered as homogeneous. Consequently, the proposed models provide only a rough estimation of the stress state in the material.

Remark 3. It was found that the change in swelling levels before and after mechanical testing was less than $0.4 \%$. Our further investigations in Loo, Le Cam, Andriyana, Robin, and Afifi (2015) have shown that even after undergoing fatigue loading (long-term cyclic loading) in air, the swelling level of rubber did not evolve significantly (less than $0.5 \%$ ). Thus, in the following model development, for the sake of simplicity, the swollen rubber is assumed to undergo mechanical deformation at constant volume.

\section{Constitutive equations}

As highlighted in the Introduction, two classical models widely used to describe Mullins effect in dry rubber are considered in the present work as the starting point: CDM model (Miehe, 1995; Simo, 1987; Miehe \& Keck, 2000; Chagnon et al., 2004) and PE model (Ogden \& Roxburgh, 1999). For this purpose, brief review on the features of each model is first presented. Subsequently, general theoretical framework for the hyperelasticity of swollen elastomers coupled with damage describing Mullins effect is derived. Within this theoretical framework, the two classical models are phenomenologically extended to account for swelling. Finally, the efficiency of each extended model in capturing Mullins effect in swollen elastomers is discussed.

\subsection{Brief review on models under consideration}

\subsubsection{CDM model}

In order to describe the softening due to Mullins effect, Simo (1987) adopted the concept of CDM. More precisely, the author proposed to multiply the classical hyperelastic strain energy of rubber $W_{0}$ with a reducing parameter $1-d$. Thus, the hyperelastic strain energy of rubber undergoing Mullins effect is given by: 

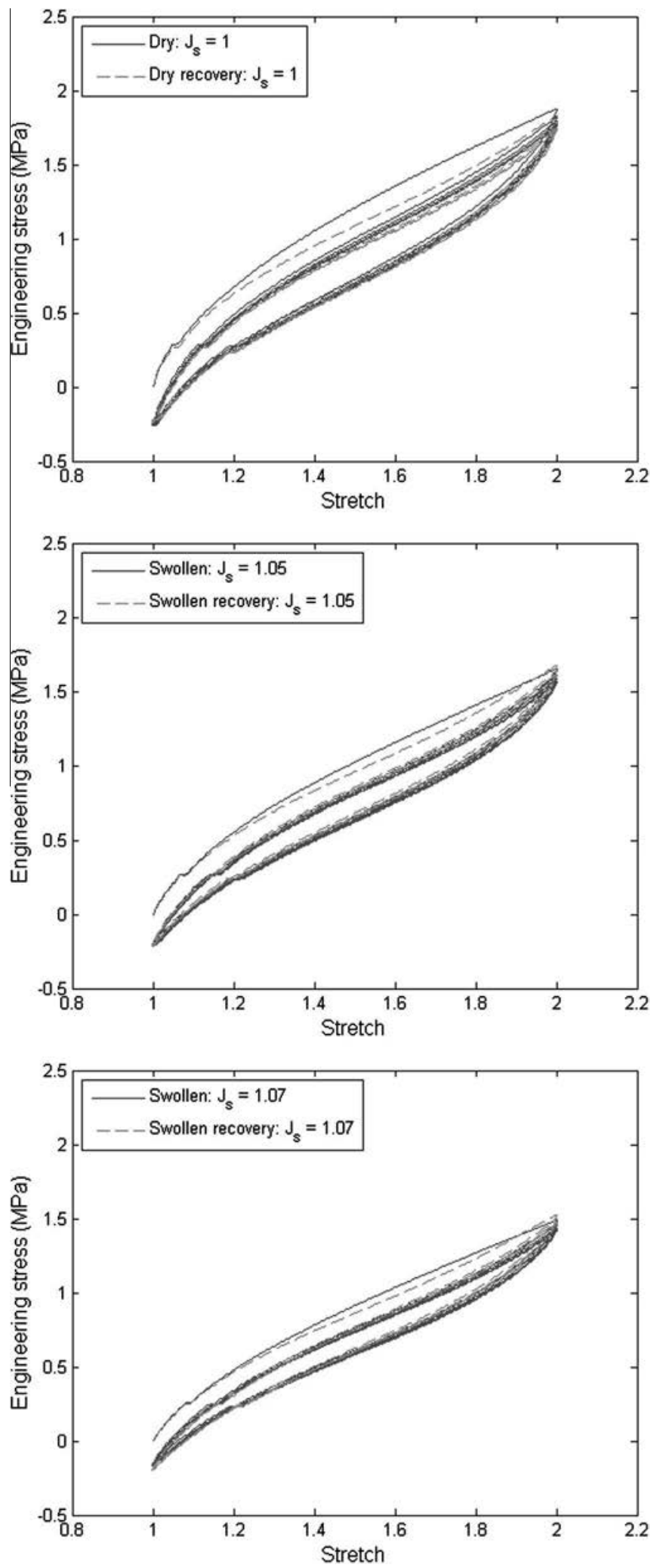

Fig. 6. Recovery of Mullins effect. (For interpretation of the references to color in this figure legend, the reader is referred to the web version of this article.) 

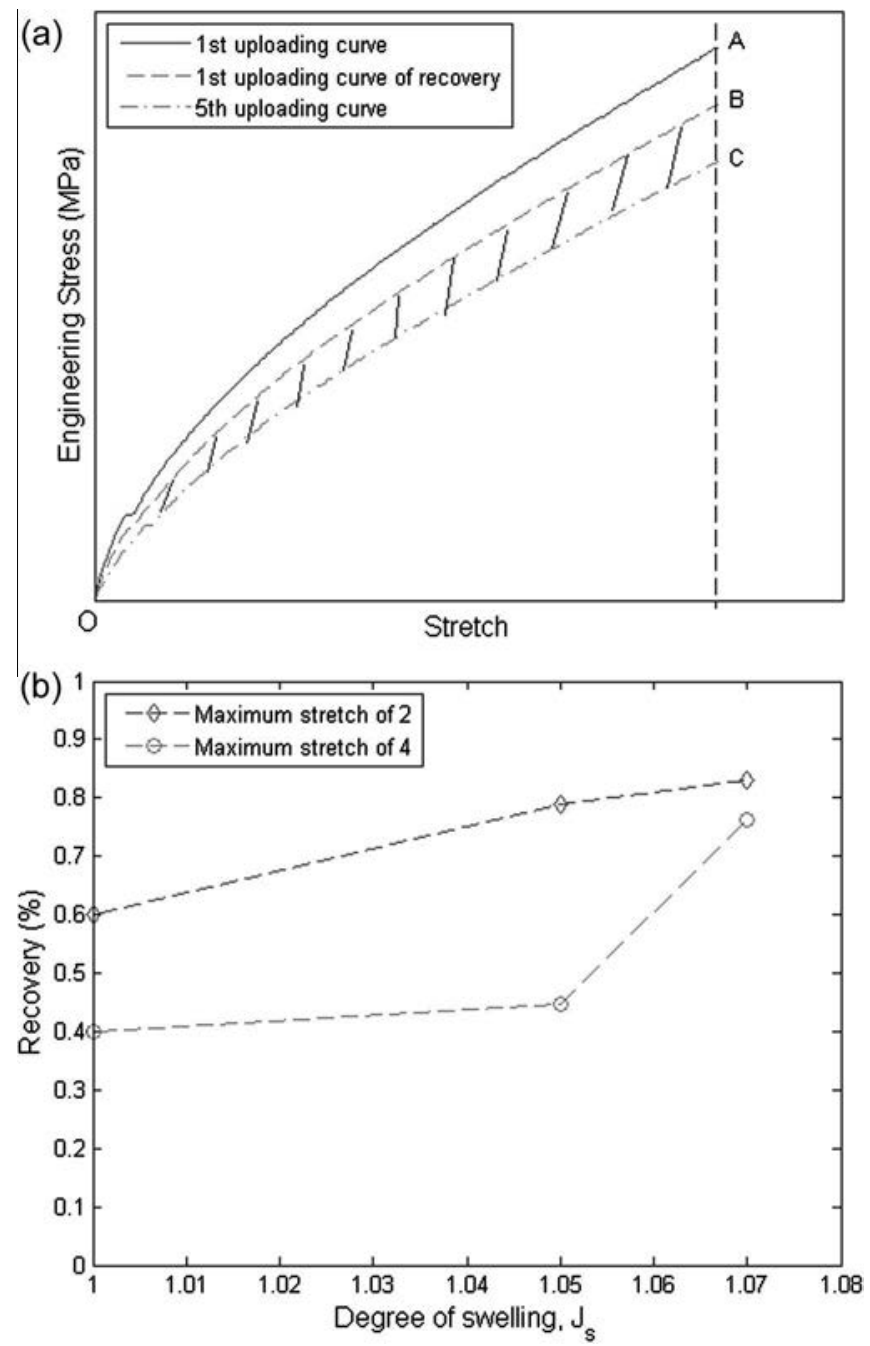

Fig. 7. (a): Graphical representation on the definition of "\% of recovery". (b) Evolution of recovery of Mullins effect as a function of degree of swelling.

$$
W=(1-d) W_{0},
$$

where $0 \leqslant d \leqslant 1$ is the damage parameter describing the internal damage in the materials due to Mullins effect. Assuming isotropy and incompressibility of material, the Cauchy stress tensor is simply given by:

$$
\boldsymbol{\sigma}=-p \mathbf{I}+2(1-d)\left[\left(\frac{\partial W_{0}}{\partial I_{1}}+I_{1} \frac{\partial W_{0}}{\partial I_{2}}\right) \mathbf{B}-\frac{\partial W_{0}}{\partial I_{2}} \mathbf{B}^{2}\right],
$$

where $p$ is a Lagrange multiplier due to incompressibility assumption and $\mathbf{I}$ is the identity tensor. $\mathbf{B}=\mathbf{F} \mathbf{F}^{\mathrm{T}}$ is the left CauchyGreen strain tensor with $\mathbf{F}$ being the deformation gradient tensor. $I_{1}$ and $I_{2}$ are the first two invariants of $\mathbf{B}$ defined by:

$$
I_{1}=\operatorname{tr} \mathbf{B} \quad I_{2}=\frac{1}{2}\left(I_{1}^{2}-\operatorname{tr} \mathbf{B}^{2}\right) .
$$

As highlighted in Diani et al. (2009), various forms of $d$ are proposed in the literature. According to Miehe (1995) and Chagnon et al. (2004), a constitutive particularization of the damage variable $d$ consistent with the second law of thermodynamics may be given by:

$$
d=d_{\infty}\left[1-\exp \left(-\frac{\alpha_{\max }}{\eta}\right)\right]
$$

where $d_{\infty}$ is the maximum possible damage and $\eta$ is the damage saturation parameter. In this damage expression, $\alpha_{\text {max }}$ is a scalar representing the maximum value of damage governing variable. For Miehe (1995), considering the "natural" driving force of damage from the second law of thermodynamics, $\alpha_{\max }$ simply corresponds to the maximum value of strain energy 
during loading history. In order to obtain a closed-form stress-stretch relationship, Chagnon et al. (2004) proposed to simplify the approach by relating $\alpha_{\max }$ to the deformation state through:

$$
\alpha_{\max }=\sqrt{\frac{I_{1 \max }}{3}}-1
$$

where $I_{1 \max }$ is the maximum value of $I_{1}$. In the following, we opt to use the approach of Chagnon et al. (2004).

\subsubsection{PE model}

The PE model was proposed by Ogden and Roxburgh (1999). It has a similar root with CDM model in the sense that the strain energy function of the material has the same form as the one given in Eq. (2) and thus, the stress by Eq. (3). However, due to the peculiar nature of the reducing parameter $1-d$, the ensuing theory is often referred to as pseudo-elasticity. Indeed, the reducing parameter is only activated during unloading and reloading to submaximum deformation. More precisely, the damage parameter $d$ is set to be zero during the loading to the deformation state never attained in the loading history and only evolves (in the range of $0 \leqslant d \leqslant 1$ ) during unloading and reloading to submaximum deformation. In this context, the authors showed that the damage variable evolves through:

$$
d=\frac{1}{r} \operatorname{erf}\left[\frac{1}{m}\left(W_{0, \max }-W_{0}\right)\right]
$$

where $r, m$ are material parameters and erf is the error function. The parameter $r$ corresponds to a measure of the extent of the damage relative to the virgin state while $m$ controls the dependence of the damage on the extent of deformation (Ogden \& Roxburgh, 1999). $W_{0, \max }$ corresponds to the maximum value of $W_{0}$ during the loading history. Note that unlike CDM model where $d$ depends exclusively on the maximum deformation state, here it is proposed that damage depends on both the current and maximum deformation states. Later on, the theory was extended by Dorfmann and Ogden (2004) to account for permanent set and by Mars (2004) to avoid an overly stiff response of the model at large deformations and to provide more flexibility in calibrating the model using experimental data.

In the following discussion, similarly to CDM model, in order to obtain a closed-form stress-stretch relationship, $\alpha_{\max }-\alpha$ will be used as damage governing variable. Thus, Eq. (7) is modified to become:

$$
d=\frac{1}{r} \operatorname{erf}\left[\frac{1}{m}\left(\alpha_{\max }-\alpha\right)\right]
$$

where $\alpha_{\max }$ is given by Eq. (7)

$$
\alpha=\sqrt{\frac{I_{1}}{3}}-1
$$

\subsection{Hyperelasticity coupled with damage for swollen elastomers}

\subsubsection{Kinematics}

In the experimental procedure, stress-free dry rubber specimens are immersed in solvent to allow isotropic swelling to occur in the material. After a desired degree of swelling is achieved, the resulting swollen specimens are subjected to various mechanical loading conditions. The corresponding procedure is illustrated in Fig. 9.

Initially, the rubber specimen is in dry state at $\mathcal{C}_{0}$ (unswollen-unstressed configuration) having a volume of $V_{0}$. It undergoes isotropic swelling characterized by the swelling part of the total deformation gradient $\mathbf{F}$, denoted $\mathbf{F}_{\mathrm{s}}$. Due to swelling, the specimen volume changes to $V_{s}$ at $\mathcal{C}_{s}$ (swollen-unstressed configuration). Recalling Remark 1, the degree of swelling, $J_{s}$, is simply defined by the ratio between the volume of swollen specimen and the volume of dry specimen, i.e.

$$
J_{s}=\frac{V_{s}}{V_{0}} .
$$

Consequently, $\mathbf{F}_{\mathrm{s}}$ can be written as a function of $J_{s}$ as follow:

$$
\mathbf{F}_{\mathrm{s}}=J_{\mathrm{s}}^{1 / 3} \mathbf{I} \text {. }
$$

The next stage of deformation consists in imposing mechanical loading to the swollen specimen. The corresponding deformation is characterized by the mechanical part of deformation gradient tensor, denoted $\mathbf{F}_{\mathrm{m}}$. Here, it is assumed that the swollen rubber undergoes deformation at constant volume, i.e. swollen rubber is incompressible. Thus, the kinematic constraint to respect during this stage of deformation is det $\mathbf{F}_{\mathrm{m}}=1$. In summary, the transformation of material from $\mathcal{C}_{0}$ to $\mathcal{C}_{t}$ (swollen-stressed configuration) can be described by:

$$
\mathbf{F}=\mathbf{F}_{\mathrm{m}} \mathbf{F}_{\mathrm{s}}=J_{\mathrm{s}}^{1 / 3} \mathbf{F}_{\mathrm{m}} .
$$



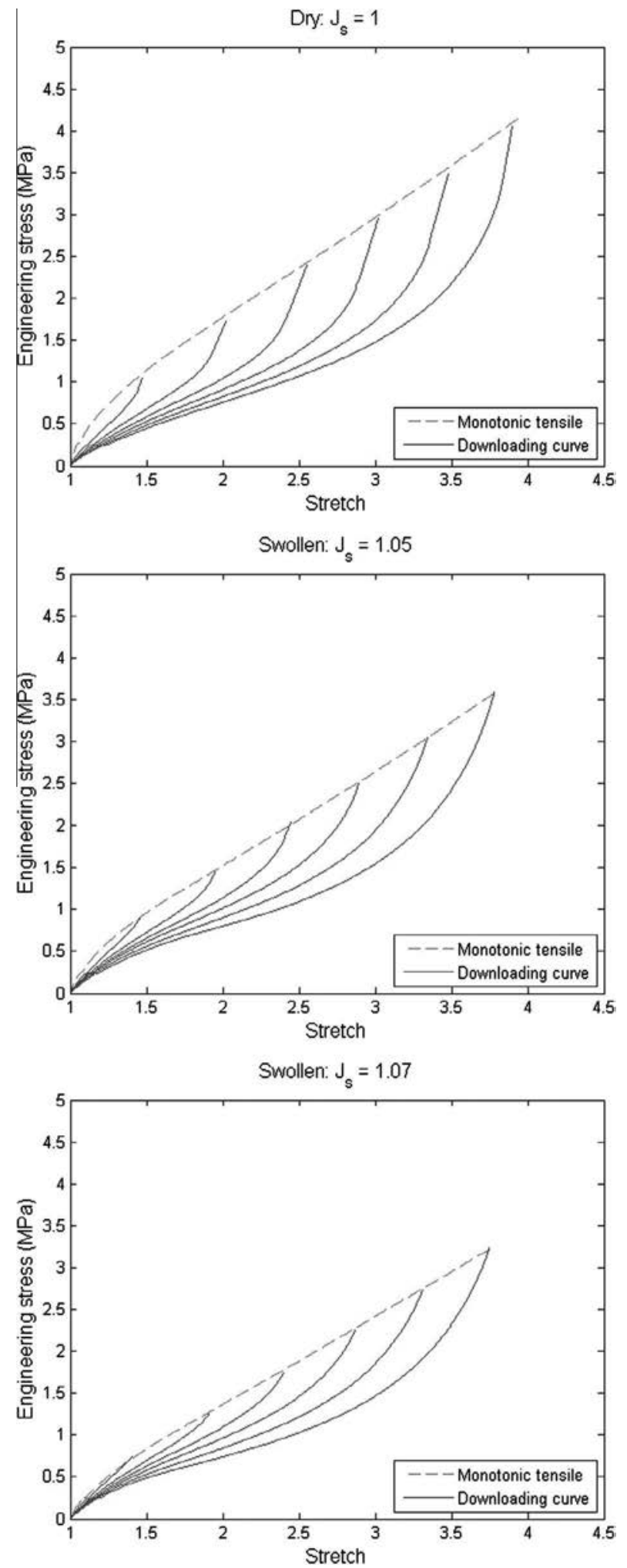

Fig. 8. Treated experimental results for modeling purpose. 


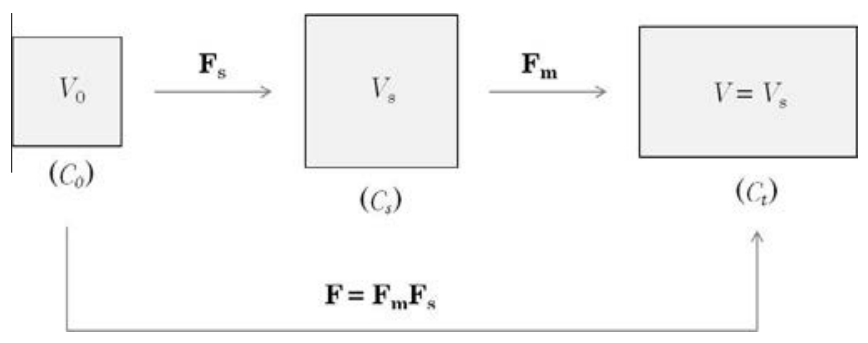

Fig. 9. Illustration of experimental procedure.

Remark 4. During the transformation from $\mathcal{C}_{0}$ to $\mathcal{C}_{t}$, rubber undergoes two types of softening:

1. Softening due to isotropic expansion (swelling) of polymeric network, i.e. transition from $\mathcal{C}_{0}$ to $\mathcal{C}_{s}$. This network expansion increases the chain separation resulting to a reduction of the secondary intermolecular bonding forces (Callister, 2007). As a consequence, the material becomes softer.

2. Softening associated with Mullins effect under cyclic mechanical loading. This softening which occurs during the transition from $\mathcal{C}_{s}$ to $\mathcal{C}_{t}$ is often regarded as essentially being caused by the fillers in rubber matrix (Holzapfel, 2000).

Since the focus of the present study is on the softening due to Mullins effect, the damage parameter $d$ is assumed to be zero at $\mathcal{C}_{s}$, i.e. before the application of mechanical loading.

Remark 5. As shown in the previous section, increasing the degree of swelling (i.e. increasing softening due to swelling) reduces the consequences of the Mullins effect. While the precise link between the above two types of softening remains unclear, it appears that swelling softening affects the material capacity to accommodate further softening under mechanical loading.

\subsubsection{Stress response}

To describe the Mullins softening in swollen elastomers, the expression of strain energy in Eq. (2) is extended:

$$
W=\tilde{W}(\mathbf{F}, d)=\hat{W}_{J_{s}}\left(\mathbf{F}_{\mathrm{m}}, d\right) .
$$

where $\hat{W}_{J_{s}}$ denotes the strain energy of swollen elastomers at a given (constant) degree of swelling, i.e. $\dot{J}_{s}=0$. In this case, for purely mechanical processes, the second law of thermodynamics becomes:

$$
\left.\mathcal{D}=J_{s}^{1 / 3} \mathbf{P}-\frac{\partial \hat{W}_{J_{s}}}{\partial \mathbf{F}_{\mathrm{m}}}\right): \dot{\mathbf{F}}_{\mathrm{m}}-\frac{\partial \hat{W}_{J_{s}}}{\partial d} \dot{d} \geqslant 0 .
$$

In this expression, $\mathcal{D}$ is the internal dissipation and $\mathbf{P}$ is the engineering stress tensor with respect to unswollen-unstrained state of the material. Considering objectivity, isotropy, incompressibility of the swollen rubber under mechanical loading and arguments of Coleman and and Gurtin (1967), it can be shown that the stress response is given by (Holzapfel, 2000):

$$
\mathbf{P}=q J_{s}^{-1 / 3} \mathbf{F}_{\mathrm{m}}^{-\mathrm{T}}+2 J_{s}^{-2 / 3}\left[\left(\frac{\partial \hat{W}_{J_{s}}}{\partial I_{1 \mathrm{~m}}}+I_{1 \mathrm{~m}} \frac{\partial \hat{W}_{J_{s}}}{\partial I_{2 \mathrm{~m}}}\right) \mathbf{F}_{\mathrm{m}}-\frac{\partial \hat{W}_{J_{s}}}{\partial I_{2 \mathrm{~m}}} \mathbf{F}_{\mathrm{m}} \mathbf{C}_{\mathrm{m}}\right],
$$

where $q$ is an arbitrary scalar (Lagrange multiplier) due to incompressibility assumption which can be determined from the equilibrium equations and appropriate boundary conditions. $\mathbf{C}_{\mathrm{m}}=\mathbf{F}_{\mathrm{m}}^{\mathrm{T}} \mathbf{F}_{\mathrm{m}}$ is the mechanical part of the right Cauchy-Green strain tensor while $I_{1 \mathrm{~m}}$ and $I_{2 \mathrm{~m}}$ are its invariants defined by:

$$
I_{1 \mathrm{~m}}=\operatorname{tr} \mathbf{C}_{\mathrm{m}} \quad I_{2 \mathrm{~m}}=\frac{1}{2}\left(I_{1 \mathrm{~m}}^{2}-\operatorname{tr} \mathbf{C}_{\mathrm{m}}^{2}\right)
$$

The Cauchy stress tensor is simply given by $\boldsymbol{\sigma}=(\operatorname{det} \mathbf{F})^{-1} \mathbf{P} \mathbf{F}^{\mathrm{T}}$ which yields to:

$$
\boldsymbol{\sigma}=q J_{s}^{-1} \mathbf{I}+2 J_{s}^{-1}\left[\left(\frac{\partial \hat{W}_{J_{s}}}{\partial I_{1 \mathrm{~m}}}+I_{1 \mathrm{~m}} \frac{\partial \hat{W}_{J_{s}}}{\partial I_{2 \mathrm{~m}}}\right) \mathbf{B}_{\mathrm{m}}-\frac{\partial \hat{W}_{J_{s}}}{\partial I_{2 \mathrm{~m}}} \mathbf{B}_{\mathrm{m}}^{2}\right] .
$$

In the case where $\hat{W}_{J_{s}}$ is expressed as a function of the principal mechanical stretches $\lambda_{\text {im }}$, defined by the ratio between the current length of swollen-stressed rubber with the one of swollen-unstressed rubber, the principal Cauchy stresses $\sigma_{i}$ can be related to the principal mechanical stretches as follow (Holzapfel, 2000): 


$$
\begin{aligned}
& \sigma_{i}=q J_{s}^{-1}+J_{s}^{-1} \lambda_{i \mathrm{~m}} \frac{\partial \hat{W}_{J_{s}}}{\partial \lambda_{i \mathrm{~m}}} \\
& i=1,2,3 \text { no sum on } i .
\end{aligned}
$$

When dealing with swollen rubbers, it is more convenient to express the engineering stress tensor with respect to swollen-unstressed state of the material $\mathcal{C}_{s}$, denoted $\hat{\mathbf{P}}$. Its principal values can be obtained using a simple relation below:

$$
\begin{aligned}
& \hat{P}_{i}=\frac{\sigma_{i}}{\lambda_{\text {im }}} \\
& i=1,2,3 \quad \text { no sum on } i .
\end{aligned}
$$

The remaining dissipation in Eq. (14) reduces to:

$$
-\frac{\partial \hat{W}_{J_{s}}}{\partial d} \dot{d} \geqslant 0
$$

which must be respected during deformation process (Andriyana, Billon, \& Silva, 2010a). In summary, two material functions have to be postulated in order to completely define the material response: the strain energy $\hat{W}_{J_{s}}$ in Eq. (13) and the evolution of damage parameter $d$ respecting inequality in Eq. (20).

Remark 6. The proposed model focuses on the Mullins effect in swollen rubbers at a given (constant) degree of swelling $J_{s}$. The general coupling problems between liquid diffusion and mechanical deformation are not considered. Indeed, the parameter $J_{S}$ is introduced merely as a means of defining the state of swelling of the elastomeric network regardless of whether or not this state is the equilibrium state with respect to the liquid absorption (Treloar, 1975).

Remark 7. For a more general case involving non-homogeneous deformation and non-uniform distribution of palm biodiesel, it is necessary to use the general theory of mixture as highlighted in Rajagopal and Tao (1995) and Baek and Pence (2011).

\subsection{Extension of classical models}

As highlighted at the end of Section 3.2.2, the form of $\hat{W}_{J_{s}}$ and the evolution of damage parameter $d$ have to be postulated. According to Treloar (1975), swelling in rubber is a purely mixing or interdiffusion process with no chemical attraction between rubber and liquid molecules. Furthermore, the only effect of swelling is to reduce the modulus in inverse proportion to the cube root of the swelling degree without changing the form of the stress-strain curves. Nevertheless, as shown in our previous work (Chai et al., 2011), deviation of Treolar's theory was observed. Hence, following Durning and Morman (1993), we postulate the following form of $\hat{W}_{J_{s}}$ :

$$
W \equiv \hat{W}_{J_{s}}\left(\lambda_{1 \mathrm{~m}}, \lambda_{2 \mathrm{~m}}, \lambda_{3 \mathrm{~m}}, d\right)=J_{s}^{-n} \cdot \bar{W}\left(\lambda_{1 \mathrm{~m}}, \lambda_{2 \mathrm{~m}}, \lambda_{3 \mathrm{~m}}, d\right)=J_{s}^{-n} \cdot(1-d) \bar{W}_{0}\left(\lambda_{1 \mathrm{~m}}, \lambda_{2 \mathrm{~m}}, \lambda_{3 \mathrm{~m}}\right)
$$

where $n>0$ is a material parameter which depends on the rubber-solvent combination (Chai et al., 2011). $\lambda_{1 \mathrm{~m}}, \lambda_{2 \mathrm{~m}}$ and $\lambda_{3 \mathrm{~m}}$ are the three principal mechanical stretches. In the following discussion, the hyperelastic strain energy of Ogden (1972) is retained. Its expression is given by:

$$
\bar{W}_{0}=\sum_{p=1}^{3} \frac{\mu_{p}}{\alpha_{p}}\left(\lambda_{1 \mathrm{~m}}^{\alpha_{p}}+\lambda_{2 \mathrm{~m}}^{\alpha_{p}}+\lambda_{3 \mathrm{~m}}^{\alpha_{p}}-3\right)
$$

where $\mu_{p}$ and $\alpha_{p}$ are material parameters. Using this form of strain energy, the expression of engineering stress with respect to the swollen-unstressed configuration $\mathcal{C}_{s}$ in Eq. (19) becomes:

$$
\hat{P}_{1}=J_{s}^{-(n+1)}(1-d)\left[\sum_{p=1}^{3} \mu_{p}\left(\lambda_{1 \mathrm{~m}}^{\alpha_{p}-1}-\lambda_{1 \mathrm{~m}}^{-\alpha_{p} / 2-1}\right)\right] .
$$

The second material function to be postulated is the damage evolution law. Considering experimental results, Mullins effect depends additionally on the swelling, i.e. damage parameter $d$ depends also on the degree of swelling $J_{s}$. In the following, simple phenomenological extensions of damage evolution describing Mullins effect which take into consideration swelling are proposed.

\subsubsection{Extended-CDM model}

A simple extension of damage parameter expression in Eq. (5) is proposed as follow:

$$
\begin{aligned}
& d \equiv \tilde{d}\left(J_{s}, \alpha_{\max }\right)=d_{\infty}\left(J_{s}\right)\left[1-\exp \left(-\frac{\alpha_{\max }}{\eta\left(J_{s}\right)}\right)\right] \\
& \alpha_{\max }=\sqrt{\frac{I_{1 \max }}{3}}-1
\end{aligned}
$$


Here, the dependence of $d$ on $J_{s}$ is assumed through the material parameters $d_{\infty}$ and $\eta$. In order to investigate the explicit dependence of $d_{\infty}$ and $\eta$ on $J_{s}$, the evolution of $d$ as a function of $\alpha_{\max }$ for different values of $J_{s}$ has to be plotted by considering the treated experimental data in Fig. 8. As detailed in Chagnon et al. (2004), for a given degree of swelling, the corresponding variable can be determined by firstly calculating the ratio between engineering stresses of two successive secondary curves in Fig. 8. The example of corresponding ratios for dry and swollen rubbers is presented in Fig. 10. As shown in this figure, the ratios are relatively constant for small strain (referred to as the proportional zone $A$ ) and decreases for larger strain level (referred to as the non-proportional zone B). According to Chagnon et al. (2004), the CDM approach for Mullins effect is based on the proportionality of stress ratios between two successive secondary curves. To this end, by taking into consideration only the proportional zone, the evolution of damage variable $d$ as a function of maximum deformation state $\alpha_{\max }$ can be obtained for dry and swollen rubbers and is presented in Fig. 11.

As shown in Fig. 11, the damage curves for dry and swollen rubbers are different. For given maximum deformation, the damage due to Mullins effect in dry rubber is higher than that in swollen one. Recalling Eq. (24), $d_{\infty}$ corresponds to the maximum possible damage while $\eta$ corresponds to the inverse of initial slope of the damage curve. Their evolution as a function of $J_{s}-1$ are estimated and plotted in Fig. 12. It appears that the corresponding dependence can be approximately described by simple linear equations as follow:

$$
\begin{aligned}
& d_{\infty}=d_{\infty, d}+a\left(J_{s}-1\right), \\
& \eta=\eta_{d}+b\left(J_{s}-1\right),
\end{aligned}
$$

where $d_{\infty, d}$ and $\eta_{d}$ are respectively the maximum possible damage and the damage saturation parameter of dry rubber while $a<0$ and $b>0$ are additional material parameters.
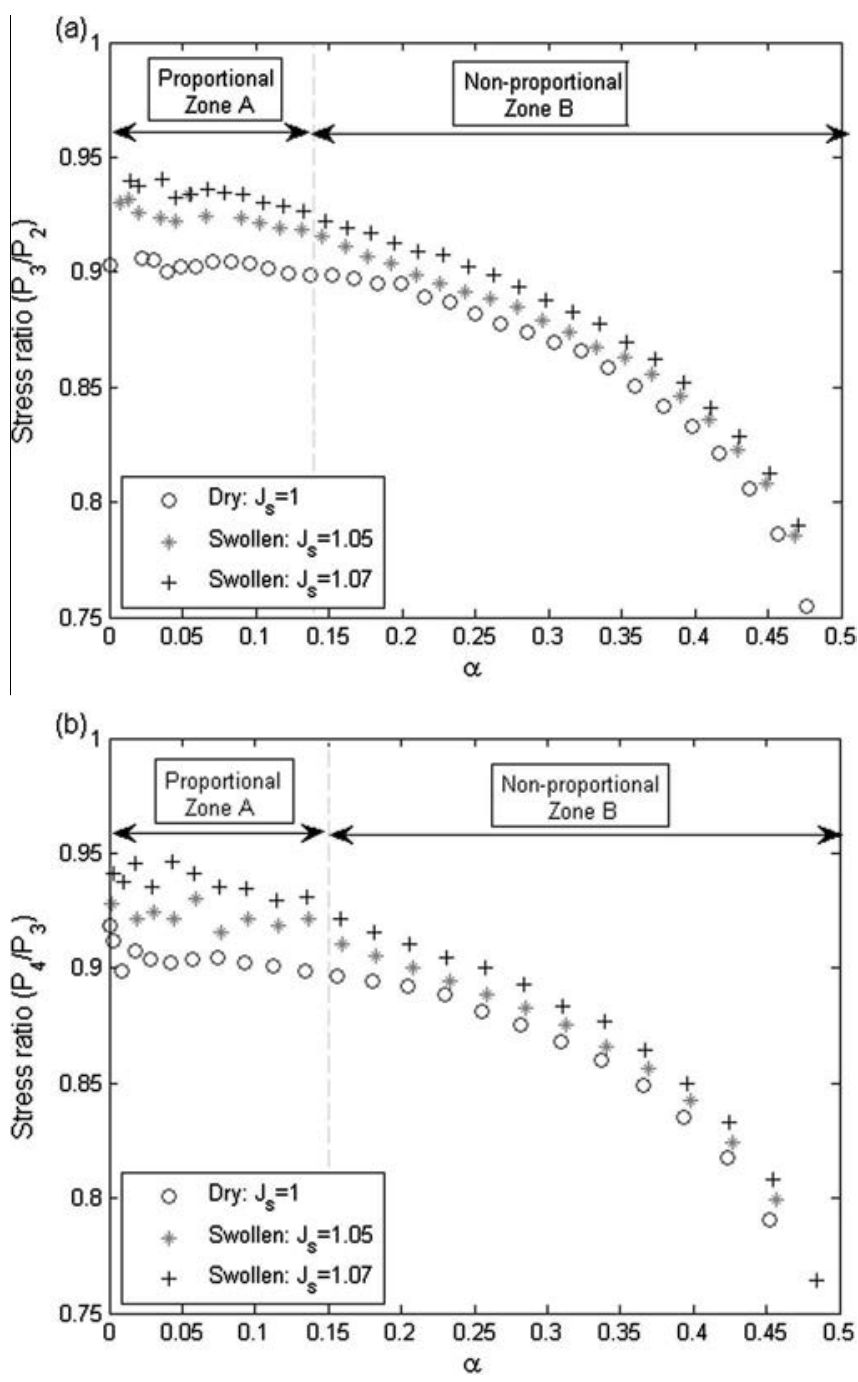

Fig. 10. Example of ratio between engineering stresses of two successive secondary curves: between the third and second secondary curves (a) and between the fourth and third secondary curves (b) as a function of damage governing variable $\alpha$. 


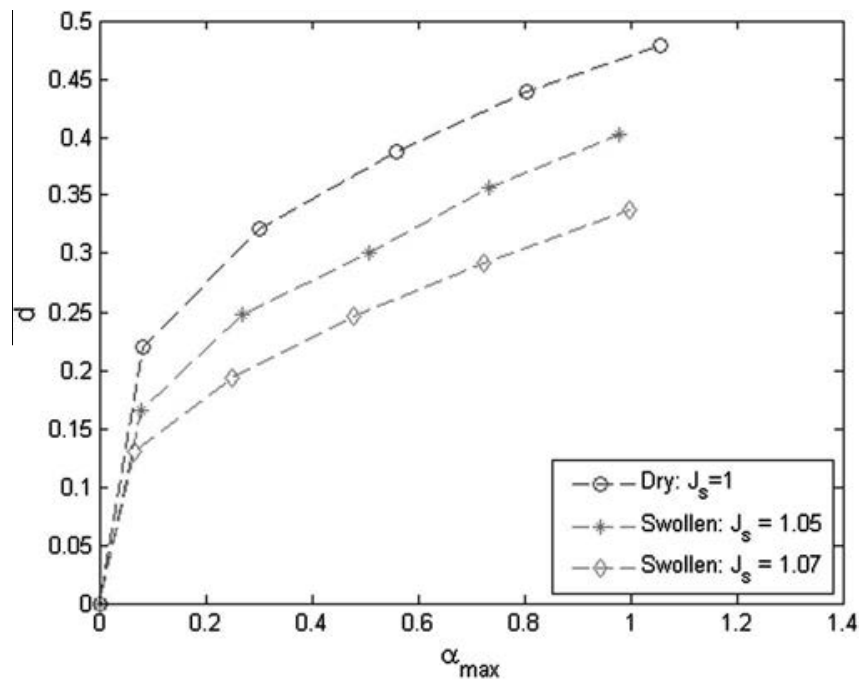

Fig. 11. Evolution of damage variable $d$ as a function of $\alpha_{\max }$ for different values of $J_{s}$.
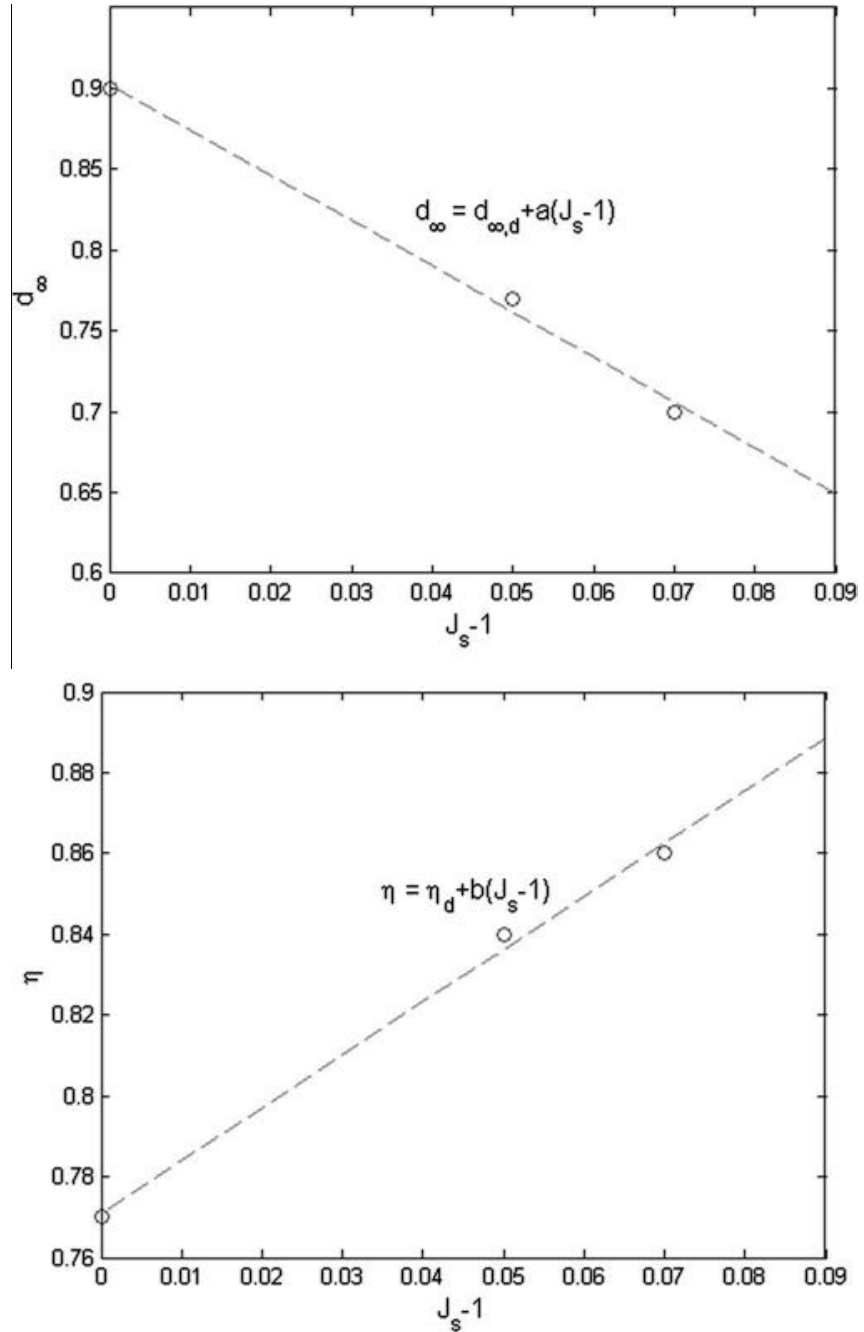

Fig. 12. Plot of estimated $d_{\infty}$ (theoretical maximum damage) and $\eta$ (damage saturation parameter) as a function of degree of swelling. 

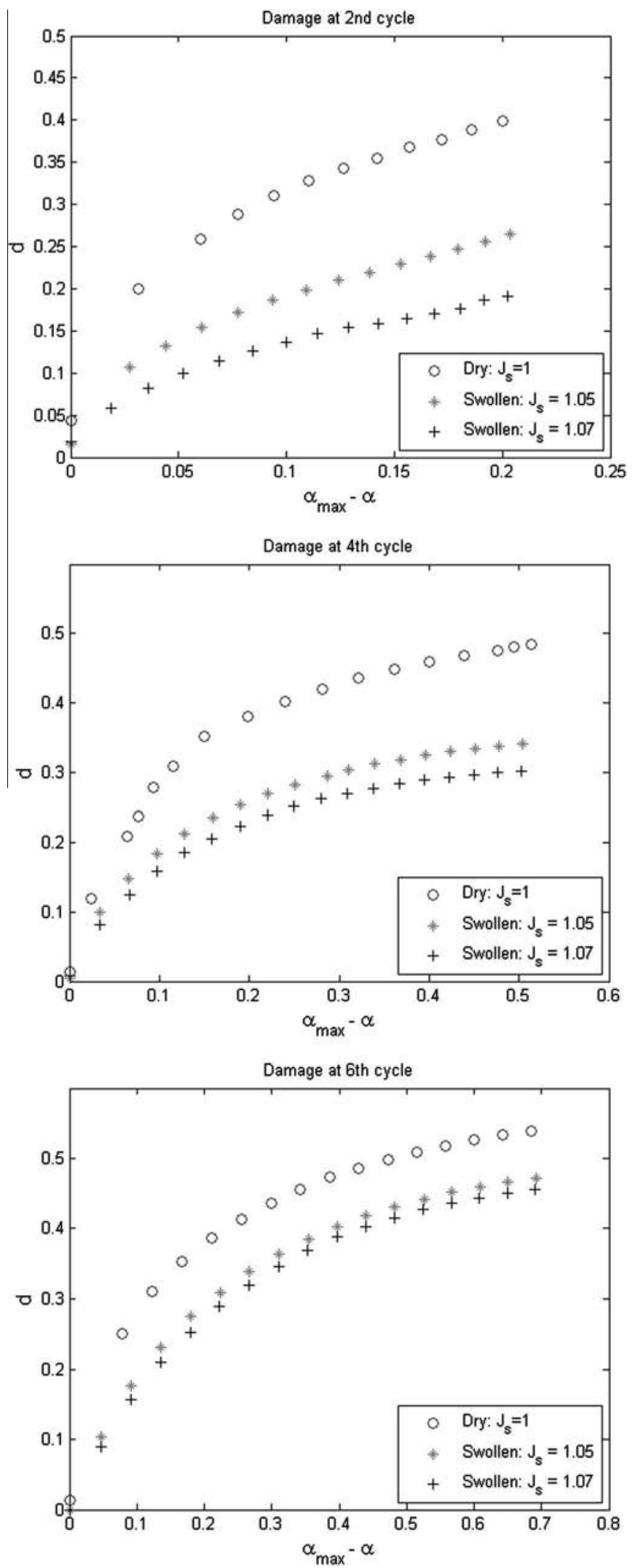

Fig. 13. Evolution of damage variable $d$ as a function of $\alpha_{\max }-\alpha$ for different values of $J_{s}$. 
In summary, the following material parameters have to be identified from the experimental data in Fig. 8: $\left(\mu_{i}, \alpha_{i}\right)_{i=1,2,3}$ for hyperelasticity, $n$ for swelling and $d_{\infty, d}, \eta_{d}, a, b$ for damage.

\subsubsection{Extended-PE model}

A simple extension of damage parameter expression in Eq. (7) is proposed as follow:

$$
\begin{aligned}
& d \equiv \hat{d}\left(J_{s}, \alpha_{\max }, \alpha\right)=\frac{1}{r\left(J_{s}\right)} \operatorname{erf}\left[\frac{1}{m\left(J_{s}\right)}\left(\alpha_{\max }-\alpha\right)\right] \\
& \alpha_{\max }=\sqrt{\frac{I_{1 \max }}{3}}-1 .
\end{aligned}
$$

Here, similarly to Eq. (24), the dependence of $d$ on $J_{s}$ is considered through the material parameters $r$ and $m$. The examples of evolution of damage $d$ as a function of $\alpha_{\max }-\alpha$ are presented in Fig. 13 where three secondary curves are considered: 2nd, 4th and 6th secondary curves. In this figure, the damage is calculated using the following equation:

$$
d=1-\frac{P_{s}}{P_{p}},
$$

where $P_{s}$ and $P_{p}$ are respectively the values of stress at secondary curve and primary curve for a given value of stretch (refer to Fig. 8). From Fig. 13, the following conclusions can be drawn:

1. Damage depends on the maximum deformation ever experienced and the current deformation state. The higher the maximum deformation, the higher the damage.

2. For a given maximum deformation, the damage in dry rubber is systematically higher than the one in swollen rubbers.

3. Higher swelling leads to a lower damage.

Recalling Eq. (7) and inspecting Fig. 13, the parameters $1 / r$ and $1 / m$ correspond respectively to the maximum value of damage and the initial slope of the damage curve. Moreover, it is clear that the maximum damage and the initial slope of the experimental damage curve depend strongly on the degree of swelling. In order to further investigate the explicit dependence of $1 / r$ and $1 / m$ on $J_{s}$, the values of inverse initial slope $m$ and inverse maximum damage $r$ are plotted as a function of $J_{s}-1$ and are presented in Fig. 14.

As shown in Fig. 14, it appears that both parameters $r$ and $m$ evolve approximately in a linear way with the degree of swelling $J_{s}$. Thus, the following linear equations may be considered to account for the dependence of $r$ and $m$ on the degree of swelling:

$$
\begin{aligned}
& r=r_{d}+\Delta r\left(J_{s}-1\right), \\
& m=m_{d}+\Delta m\left(J_{s}-1\right),
\end{aligned}
$$

where $r_{d}, m_{d}, \Delta r$ and $\Delta m$ are material parameters. Note that $r_{d}$ and $m_{d}$ correspond to the value of $r$ and $m$ for dry rubber.

In summary, the following material parameters have to be identified from the experimental data in Fig. 8: $\left(\mu_{i}, \alpha_{i}\right)_{i=1,2,3}$ for hyperelasticity, $n$ for swelling and $r_{d}, m_{d}, \Delta r, \Delta m$ for damage.

Remark 8. While the extension of PE-Model presented here follows the work of Chai et al. (2013b), our starting point on the general derivation of the model is different. Indeed, Chai et al. (2013b) developed a model based on the consideration that swollen elastomers can be directly treated as isotropic, incompressible and hyperelastic material. Thus, the derivation of the model follows exactly the one of classical hyperelastic material with additional dependence of strain energy on the degree of swelling. In our present approach, our starting point is the split of deformation gradient tensor into mechanical and swelling parts. Thereby, capturing the experimental procedure where stress-free rubber specimens are initially immersed in solvent before undergoing mechanical loading.

\section{Simulation}

In this Section, the comparison between the extended models and experimental data is presented. To this end, strategies in the identification of material parameters involved in the simulation are firstly described. Subsequently, the advantage and limitation of each extended model in capturing Mullins effect in swollen rubbers is discussed and perspectives are drawn.

\subsection{Identification of material parameters}

For the extended-CDM model, it is first necessary to identify simultaneously material parameters related to dry rubber as follow: $\mu_{1}, \mu_{2}, \mu_{3}, \alpha_{1}, \alpha_{2}, \alpha_{3}, d_{\infty, d}$ and $\eta_{d}$. For this purpose, the experimental data of dry rubber provided in Figs. 8 and 11 

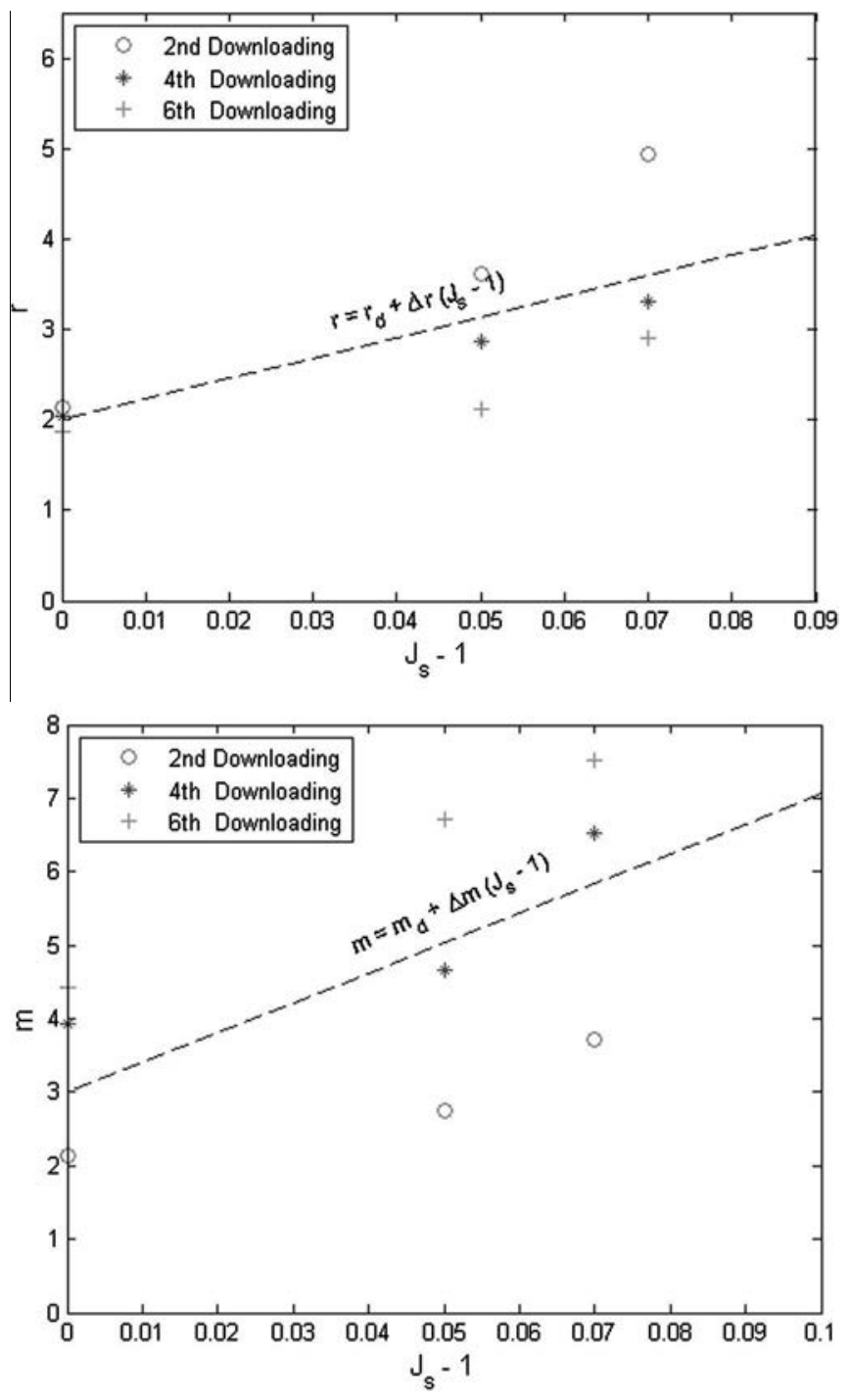

Fig. 14. Plot of $r$ and $m$ as a function of degree of swelling.

Table 1

Value of material parameters used in extended-CDM model.

\begin{tabular}{ll}
\hline Parameter & Value \\
\hline$\mu_{1}$ & $0.74 \mathrm{MPa}$ \\
$\mu_{2}$ & $-6.39 \mathrm{MPa}$ \\
$\mu_{3}$ & $0.04 \mathrm{MPa}$ \\
$\alpha_{1}$ & 2.12 \\
$\alpha_{2}$ & -0.06 \\
$\alpha_{3}$ & 5.46 \\
$n$ & 4.31 \\
$d_{\infty, d}$ & 0.9 \\
$a$ & -2.8 \\
$\eta_{d}$ & 0.77 \\
$b$ & 1.3 \\
\hline
\end{tabular}


Table 2

Value of material parameters used in extended-PE model.

\begin{tabular}{ll}
\hline Parameter & Value \\
\hline$\mu_{1}$ & $0.81 \mathrm{MPa}$ \\
$\mu_{2}$ & $-0.01 \mathrm{MPa}$ \\
$\mu_{3}$ & $0.36 \mathrm{MPa}$ \\
$\alpha_{1}$ & 1.66 \\
$\alpha_{2}$ & -4.90 \\
$\alpha_{3}$ & 2.38 \\
$n$ & 9.50 \\
$r_{d}$ & 1.80 \\
$\Delta r$ & 4 \\
$m_{d}$ & 4 \\
$\Delta m$ & 20 \\
\hline
\end{tabular}
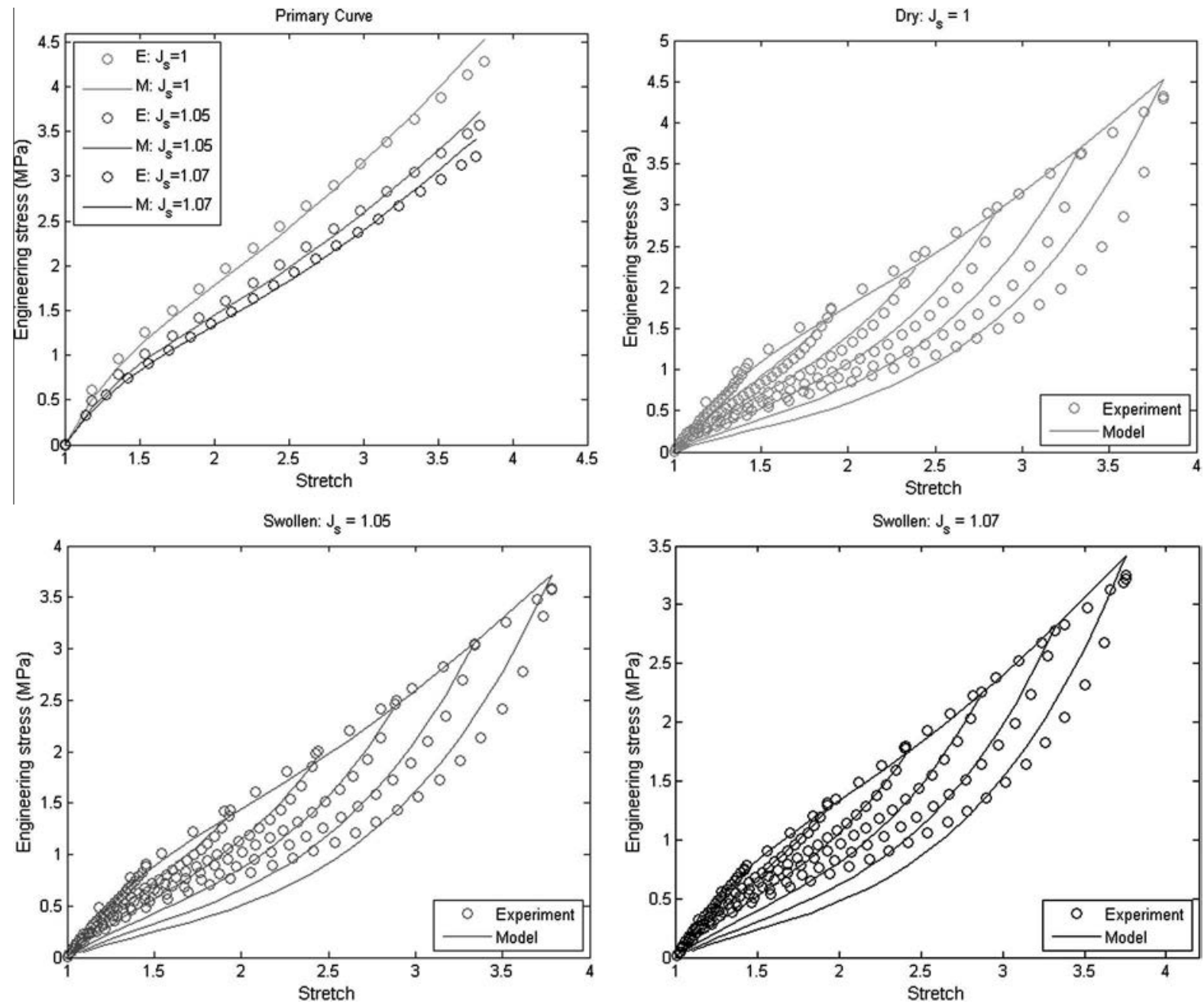

Fig. 15. Comparison between extended-CDM model and experimental data.

are considered. The physical constraints to be respected are $0<d_{\infty, d}<1$ and $\eta_{d}>0$. Moreover, in comparison with the linear theory, the following consistency condition must be fulfilled for hyperelastic-related parameters (Holzapfel, 2000):

$$
\mu_{1} \alpha_{1}>0 \quad \mu_{2} \alpha_{2}>0 \quad \mu_{3} \alpha_{3}>0 .
$$

The remaining swelling parameters $a, b$ and $n$ are identified by considering the swollen rubber data in Figs. 8 and 11 using the values of $\mu_{1}, \mu_{2}, \mu_{3}, \alpha_{1}, \alpha_{2}, \alpha_{3}, d_{\infty, d}$ and $\eta_{d}$ obtained from previous identification. The following physical constraints 

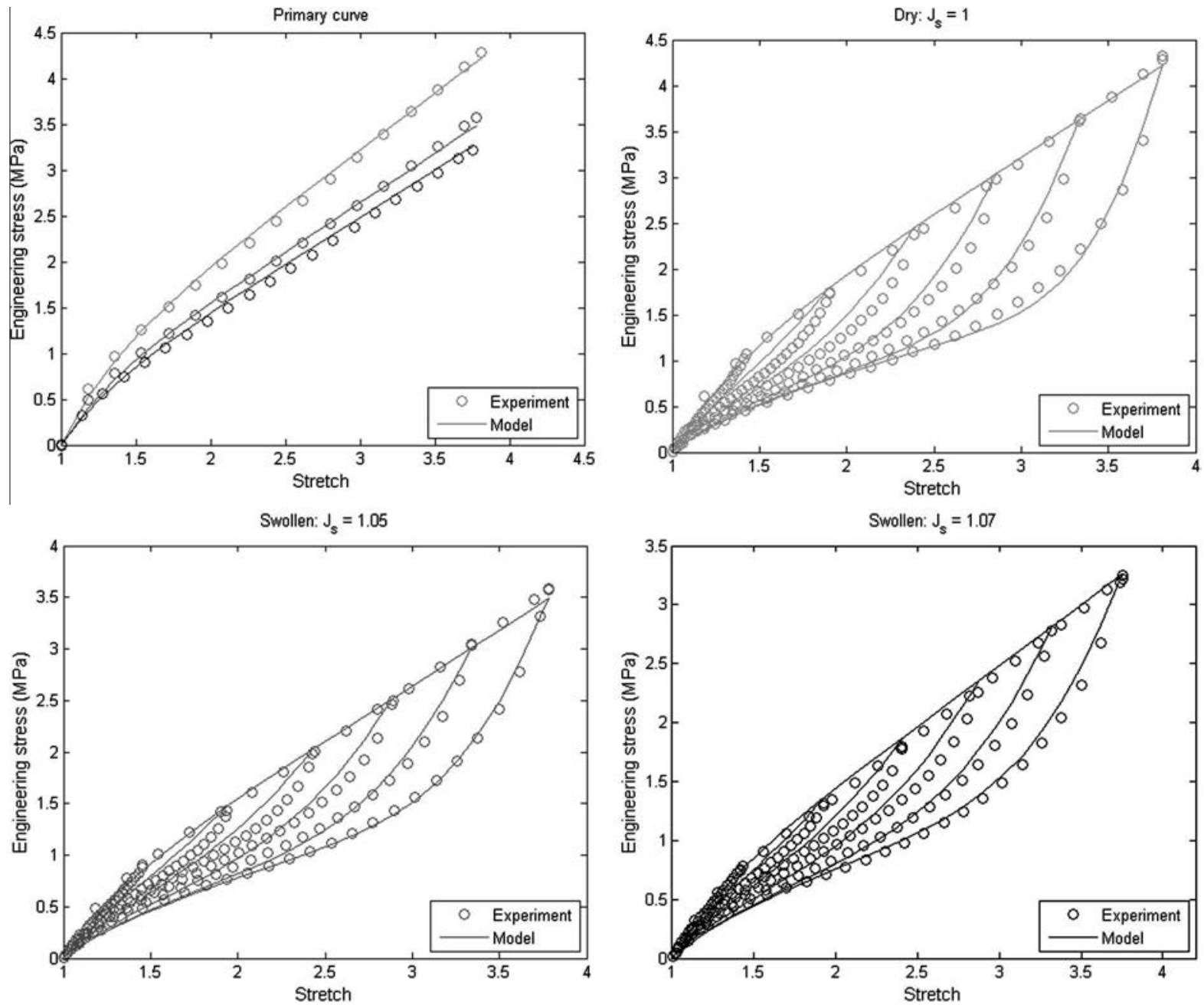

Fig. 16. Comparison between extended-PE model and experimental data.

are imposed: $a<0, b>0$ and $n>0$. Finally, the following parameters are obtained and used for simulation purpose using extended-CDM model, they are tabulated in Table 1.

For the extended-PE model, the identification process is relatively simpler due to the nature of the model itself. Indeed, when using PE model, the hyperelastic-related parameters can be identified separately from damage and swelling parameters. More precisely, $\mu_{1}, \mu_{2}, \mu_{3}, \alpha_{1}, \alpha_{2}$ and $\alpha_{3}$ can be fitted directly from the primary (monotonic tensile) curve of dry rubber in Fig. 8 without involving any damage variable. In this process, the restriction described in Eq. (29) is also imposed. Using the obtained values, the parameter $n>0$ can be estimated by fitting the primary curves of swollen rubbers in Fig. 8.

The damage parameters in dry rubber $r_{d}$ and $m_{d}$ are obtained by fitting the set of secondary (unloading) curves of dry rubber in Fig. 8. For swollen rubbers, using all parameters obtained previously, the parameters $\Delta r$ and $\Delta m$ are estimated by fitting simultaneously all sets of secondary curves of swollen rubbers in Fig. 8. Their values are subsequently adjusted by trial and error so that they fall within the range as predicted in Fig. 14. Finally, the following parameters are obtained and used for simulation purpose using extended-PE model, they are tabulated in Table 2.

\subsection{Comparison with experimental data}

The comparison between experimental data and model predictions are depicted in Figs. 15 and 16 for extended-CDM and extended-PE models respectively. For each model, four graphs are provided. The first graph corresponds to the uploading (monotonic tensile) curves while the remaining three graphs to the complete uploading-reloading curves. In general, both model capture the general feature of stress-softening due to Mullins effect. However, the superiority of extended-PE model over extended-CDM model is clearly shown. Indeed, the extended-CDM model is unable to predict correctly the unloading 
curves in dry and swollen rubbers. As reported by Chagnon et al. (2004) and Diani et al. (2009), the limited performance of the CDM model is mainly due to the following two reasons:

1. The existence of strain hardening phenomena in the rubber when it is stretched (see Fig. 10 for non-proportional area), requires an enhanced damage evolution law which enables the model to be effective in both proportional and non-proportional area.

2. Damage is assumed to depend only on the maximum deformation state in loading history. This assumption differs from the occurrence of damage in reality when the current deformation is equally essential and should be taken into account.

As illustrated in Fig. 16, the modeling results using the extended-PE appear to be in good agreement with experimental data. Both uploading and unloading curves in dry and swollen rubbers are well-described. The dependence of Mullins effect on swelling is well-captured. Despite the fact that the extended-PE model successfully reproduces the experimental observation, especially for the unloading responses, Kazakevičiūtè-Makovska (2007) and Diani et al. (2009) exemplified some doubts on the model by presenting the contrast between experimental data and theoretical result of the softening function. This contrast arises from the basic assumption, required by the second law of thermodynamics, that softening function is increasing monotonously with the deformation range. However, it appears that the experimental data do not follow the above assumption.

To close the discussion, it is to note that while both models appear to capture the general features of Mullins effect in swollen rubber, the precise link between the softening due to mechanical loading and the one due to swelling remains unclear. More precisely, since network expansion due to swelling can also be regarded as mechanical loading, Mullins effect might already take place during the swelling process. Thus, the assumption that the damage parameter $d$ is zero at $\mathcal{C}_{s}$ as stated in Remark 4 needs to be investigated in further works.

\section{Conclusion}

In this paper, the mechanical response of dry and swollen rubbers were addressed. More precisely, the focus was laid on the Mullins effect classically observed in rubber under cyclic loading conditions. The nature of Mullins effect in both dry and swollen rubbers were investigated through a set of mechanical tests. It was found that Mullins effect is smaller in the case of swollen rubber.

Based on the experimental investigations, simple phenomenological extensions of classical models for Mullins effect were proposed. Two models were considered: Continuum Damage Mechanics (CDM) model and Pseudo-Elastic (PE) model. Results showed that both models capture the general features of Mullins effect in dry and swollen rubbers. Due to the nature of the model, the extended-PE model appears to give a more satisfactory prediction than the extended-CDM model.

\section{Acknowledgments}

The authors greatly appreciate the financial support from the Ministry of Higher Education Malaysia through High Impact Research Grant HIR-MOHE D000008-16001.

\section{References}

Andriyana, A., Billon, N., \& Silva, L. (2010a). Mechanical response of a short fiber-reinforced thermoplastic: Experimental investigation and continuum mechanical modeling. European Journal of Mechanics A - Solids, 29, 1065-1077.

Andriyana, A., Saintier, N., \& Verron, E. (2010b). Configurational mechanics and critical plane approach: Concept and application to fatigue failure analysis of rubberlike materials. International Journal of Fatigue, 32(10), 1627-1638.

Andriyana, A., Chai, A. B., Verron, E., \& Johan, M. R. (2012). Interaction between diffusion of palm biodiesel and large strain in rubber: Effect on stresssoftening during cyclic loading. Mechanics Research Communications, 43, 80-86.

Arruda, E. M., \& Boyce, M. C. (1993). A three dimensional constitutive model for the large stretch behavior of rubber elastic materials. Journal of the Mechanics and Physics of Solids, 41(2), :389-412.

Baek, S., \& Pence, T. J. (2011). Inhomogeneous deformation of elastomer gels in equilibrium under saturated and unsaturated conditions. Journal of the Mechanics and Physics of Solids, 59(3), 561-582.

Beatty, M., \& Krishnaswamy, S. (2000). A theory of stress-softening in incompressible isotropic materials. Journal of the Mechanics and Physics of Solids, 48, $1931-1965$.

Bergström, J. S., \& Boyce, M. C. (1998). Constitutive modeling of the large strain time-dependent behavior of elastomers. Journal of the Mechanics and Physics of Solids, 46(5), :931-954.

Bouasse, H., \& Carriére, Z. (1903). Courbes de traction du caoutchouc vulcanisé. Annales de la Faculté des Sciences de Toulouse, 5, 257-283.

Callister, W. D. (2007). Materials science and engineering: An introduction. John Wiley \& Sons.

Chagnon, G., Verron, E., Gornet, L., Marckmann, G., \& Charrier, P. (2004). On the relevance of continuum damage mechanics as applied to the Mullins effect in elastomers. Journal of the Mechanics and Physics of Solids, 52(7), :1627-1650.

Chagnon, G., Verron, E., Marckmann, G., \& Gornet, L. (2006). Development of new constitutive equations for the Mullins effect in rubber using the network alteration theory. International Journal of Solids and Structures, 43(22-23), 6817-6831.

Chai, A. B., Andriyana, A., Verron, E., \& Johan, M. R. (2013a). Mechanical characteristics of swollen elastomers under cyclic loading. Materials \& Design, 44, 566-572.

Chai, A. B., Andriyana, A., Verron, E., \& Johan, M. R. (2013b). Mullins effect in swollen rubber: Experimental investigation and constitutive modeling. Polymer Testing, 32, 748-759.

Chai, A. B., Andriyana, A., Verron, E., Johan, M. R., \& Haseeb, A. S. M. A. (2011). Development of a compression test device for investigating interaction between diffusion of biodiesel and large deformation in rubber. Polymer Testing, 30, 867-875. 
Ch'ng, S. Y., Andriyana, A., Verron, E., Kahbasi, O., \& Ahmad, R. (2013). Development of a novel experimental device to investigate swelling of elastomers in biodiesel undergoing multiaxial large deformation. Experimental Mechanics, 53, 1323-1332.

Coleman, B. D., \& Gurtin, M. E. (1967). Thermodynamics with internal state variables. Defense Technical Information Center.

Diani, J., Brieu, M., \& Gilormini, P. (2006). Observation and modeling of the anisotropic visco-hyperelastic behavior of a rubberlike material. International Journal of Solids and Structures, 43, 3044-3056.

Diani, J., Fayolle, B., \& Gilormini, P. (2009). A review on the Mullins effect. European Polymer Journal, 45(3), 601-612.

Dorfmann, A., \& Ogden, R. W. (2004). A constitutive model for the Mullins effect with permanent set in particle-reinforced rubber. International Journal of Solids and Structures, 41, 1855-1878.

Durning, C. J., \& Morman, K. Jr., (1993). Nonlinear swelling of polymer gels. The Journal of Chemical Physics, 98(5), :4275-4293.

Flory, P. J. (1953). Principles of polymer chemistry. Cornell Univiversity Press.

Freund, M., Lorenz, H., Juhre, D., Ihlemann, J., \& Klüppel, M. (2011). Finite element implementation of a microstructure-based model for filled elastomers. International Journal of Plasticity, 27, 902-919.

Govindjee, S., \& Simo, J. C. (1991). A micro-mechanical continuum mechanical model for carbon black filled rubbers incorporating Mullins effect. Journal of the Mechanics and Physics of Solids, 39, 87-112.

Harwood, J. A. C., \& Payne, A. R. (1967). Stress softening in natural rubber vulcanizates. IV. Unfilled vulcanizates. Rubber Chemistry and Technology, 40, $840-848$.

Haseeb, A. S. M. A., Jun, T. S., Fazal, M. A., \& Masjuki, H. H. (2011). Degradation of physical properties of different elastomers upon exposure to palm biodiesel. Energy, 36(3), :1814-1819.

Holzapfel, G. A. (2000). Nonlinear solid mechanics. A continuum approach for engineering. Wiley.

Huntley, H. E., Wineman, A. S., \& Rajagopal, K. R. (1996). Chemorheological relaxation, residual stress and permanent set arising in radial deformation of an elastomeric hollow sphere. Mathematics and Mechanics of Solids, 1, 267-299.

Huntley, H. E., Wineman, A. S., \& Rajagopal, K. R. (1997). Stress softening, strain localization and permanent set in the circumferential shear of an incompressible elastomeric cylinder. IMA Journal of Applied Mathematics, 59, 309-338.

Itskov, M., Ehret, A., Kazakevičiūtè-Makovska, R., \& Weinhold, G. (2010). Experimental observation of the deformation induced anisotropy of the Mullins effect in rubber. Zeitschrift für Angewandte Mathematik und Mechanik, 90, 370-386.

Itskov, M., Haberstroh, E., Ehret, A., \& Vohringer, M. (2006). Experimental observation of the deformation induced anisotropy of the Mullins effect in rubber. Kautschuk, Gummi, Kunststoffe, 59, 93-96.

Jayed, M. H., Masjuki, H. H., Kalam, M. A., Mahlia, T. M. I., Husnawan, M., \& Liaquat, A. M. (2011). Prospects of dedicated biodiesel engine vehicles in Malaysia and Indonesia. Renewable \& Sustainable Energy Reviews, 15, 220-235.

Kazakevičiūtè-Makovska, R. (2007). Experimentally determined properties of softening functions in pseudo-elastic models of the Mullins effect. International Journal of Solids and Structures, 44, 4145-4157.

Killian, H. G., Strauss, M., \& Hamm, W. (1994). Universal properties in filler-loaded rubbers. Rubber Chemistry and Technology, 67, 1-16.

Klüppel, M., \& Schramm, M. A. (9:742-754). A generalized tube model of rubber elasticity and stress softening of filler reinforced elastomer systems. Macromolecular Theory and Simulations, 9, 742-754.

Laraba-Abbes, F., Ienny, P., \& Piques, R. (2003). A new tailor-made methodology for the mechanical behaviour analysis of rubber-like materials: II. Application to the hyperelastic behaviour characterization of a carbon-black filled natural rubber vulcanizate. Polymer, 44, 821-840.

Lin, W.-C., Fan, W., Marcellan, A., Hourdet, D., \& Creton, C. (2010). Large strain and fracture properties of poly(dimethylacrylamide)/silica hybrid hydrogels. Macromolecules, 43(5), :2554-2563.

Lion, A. (1997). On the large deformation behaviour of reinforced rubber at different temperatures. Journal of the Mechanics and Physics of Solids, 45(11-12), $1805-1834$.

Loo, M. S., Le Cam, J.-B., Andriyana, A., Robin, E., \& Afifi, A. M. (2015). Fatigue of swollen elastomers. International Journal of Fatigue, 74, $132-141$.

Machado, G., Chagnon, G., \& Favier, D. (2012). Induced anisotropy by the Mullins effect in filled silicone rubber. Mechanics of Materials, 50, 70-80.

Marckmann, G., Verron, E., Gornet, L., Chagnon, G., Charrier, P., \& Fort, P. (2002). A theory of network alteration for the Mullins effect. Journal of the Mechanics and Physics of Solids, 50, 2011-2028.

Mars, W. V. (2004). Evaluation of a pseudo-elastic model for the Mullins effect. Tire Science and Technology, 32, 120-145.

Mars, W. V., \& Fatemi, A. (2002). A literature survey on fatigue analysis approaches for rubber. International Journal of Fatigue, 24, 949-961.

Miehe, C. (1995). Discontinuous and continuous damage evolution in Ogden-type large strain elastic materials. European Journal of Mechanics - A/Solids, 14, $697-720$.

Miehe, C., \& Keck, J. (2000). Superimposed finite elasticviscoelastic plastoelastic stress response with damage in filled rubbery polymers. Experiments, modelling and algorithmic implementation. Journal of the Mechanics and Physics of Solids, 48(2), 323-365.

Mullins, L. (1948). Effect of stretching on the properties of rubber. Rubber Chemistry and Technology, 21, 281-300.

Mullins, L., \& Tobin, N. R. (1957). Theoretical model for the elastic behaviour of filler reinforced vulcanized rubbers. Rubber Chemistry and Technology, 30, $551-571$.

Ogden, R. W. (1972). Large deformation isotropic elasticity-on the correlation of theory and experiment for incompressible rubberlike solids. Proceedings of the Royal Society of London. Series A, 326(1567), :565-584.

Ogden, R. W., \& Roxburgh, D. G. (1999). A pseudo-elastic model for the Mullins effect in filled rubber. Proceedings of the Royal Society A-Mathematical Physics, 455(1988), 2861-2877.

Qi, H. J., \& Boyce, M. C. (2004). Constitutive model for stretch-induced softening of the stress-stretch behavior of elastomeric materials. Journal of the Mechanics and Physics of Solids, 52, 2187-2205.

Rajagopal, K. R., \& Tao, L. (1995). Mechanics of mixtures. Series on advances in mathematics for applied sciences (Vol. 35). World Scientific Pub.

Selvadurai, A. S. P., \& Yu, Q. (2006). Constitutive modelling of a polymeric material subjected to chemical exposure. International Journal of Plasticity, 22 $1089-1122$.

Simo, J. C. (1987). On a fully three-dimensional finite-strain viscoelastic damage model: formulation and computational aspects. Computer Methods in Applied Mechanics and Engineering, 60, 5153-5173.

Trabelsi, A., Albouy, P. A., \& Rault, J. (2003). Crystallization and melting processes in vulcanized stretched natural rubber. Macromolecules, 36(20), 7624-7639.

Treloar, L. R. G. (1975). The physics of rubber elasticity. London: Oxford University Press.

Webber, R., Creton, C., Brown, H., \& Gong, J. (2007). Large strain hysteresis and Mullins effect of tough double-network hydrogels. Macromolecules, 40, 2919-2927.

Wineman, A. S., \& Huntley, H. E. (1994). Numerical simulation of the effect of damaged induced softening on the inflation of a circular rubber membrane. International Journal of Solids and Structures, 31, 3295-3313. 